\title{
Enantioselective Synthesis of Axially Chiral Anilides through Rhodium-Catalyzed [2+2+2] Cycloaddition of 1,6-Diynes with Trimethylsilylynamides
}

\author{
Ken Tanaka,* Kenzo Takeishi, and Keiichi Noguchi \\ Department of Applied Chemistry, Graduate School of Engineering, and Instrumentation \\ Analysis Center, Tokyo University of Agriculture and Technology, Koganei, Tokyo 184- \\ 8588, Japan
}

\section{General}

Anhydrous $\mathrm{CH}_{2} \mathrm{Cl}_{2}$ (No. 27,099-7) was obtained from Aldrich and used as received. Modified-BINAP ligands (tol-BINAP, xyl-BINAP, $\mathrm{H}_{8}$-BINAP, xyl- $\mathrm{H}_{8}$-BINAP, and Segphos) were obtained from Takasago International Corporation. All other reagents were obtained from commercial sources and used as received. All reactions were carried out under an atmosphere of argon or nitrogen in oven-dried glassware with magnetic stirring. Diynes $\mathbf{1 a},{ }^{1} \mathbf{1 e},{ }^{2}$ and $\mathbf{1 \mathbf { f } ^ { 3 }}{ }^{\text {were }}$ prepared according to literatures.

\section{Synthesis of Ynamides}

General Procedure. KHMDS $(0.5 \mathrm{M}$ in toluene, $0.62 \mathrm{~mL}, 0.310 \mathrm{mmol})$ was added to a solution of $(37.7 \mathrm{mg}, 0.178 \mathrm{mmol})$ in toluene $(15 \mathrm{~mL})$ under argon at $-30{ }^{\circ} \mathrm{C}$. After the mixture was allowed to $\mathrm{rt}$, phenyl(trimethylsilylethynyl)iodonium triflate $(159.0 \mathrm{mg}, 0.353$ mmol) was added in four portions. The resulting mixture was stirred for $15 \mathrm{~h}$ at $\mathrm{rt}$ and filtered through a plug of silica gel. Purification by silica gel column chromatography (hexane:EtOAc $=40: 1)$ gave $\mathbf{2 a}(43.0 \mathrm{mg}, 0.140 \mathrm{mmol}, 78 \%$ yield $)$.

$N$-Benzyl- $N$-(trimethylsilanylethynyl)benzamide (2a). ${ }^{4}$

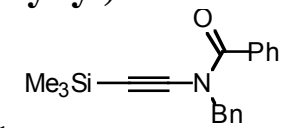

Yellow solid; Mp 38-39 ${ }^{\circ} \mathrm{C} ;{ }^{1} \mathrm{H}$ NMR $\left(\mathrm{CDCl}_{3}, 300 \mathrm{MHz}\right) \delta 7.78-7.88(\mathrm{~m}, 2 \mathrm{H})$, 7.28-7.56 (m, 8H), $4.86(\mathrm{~s}, 2 \mathrm{H}),-0.03(\mathrm{~s}, 9 \mathrm{H}) ;{ }^{13} \mathrm{C} \mathrm{NMR}\left(\mathrm{CDCl}_{3}, 75 \mathrm{MHz}\right) \delta 170.4,135.9$, $133.3,131.4,128.88,128.86,128.4,128.0,127.5,97.9,75.5,52.7,-0.3$.

$N$-Butyl- $N$-(trimethylsilanylethynyl)benzamide (2b).

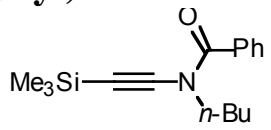

Yellow oil; IR (neat); 2900, 2150, 1670, 1270, 840, $700 \mathrm{~cm}^{-1} ;{ }^{1} \mathrm{H}$ NMR $\left(\mathrm{CDCl}_{3}, 300\right.$ $\mathrm{MHz}) \delta 7.73-7.85(\mathrm{~m}, 2 \mathrm{H}), 7.32-7.52(\mathrm{~m}, 3 \mathrm{H}), 3.69(\mathrm{t}, J=7.2 \mathrm{~Hz}, 2 \mathrm{H}), 1.75$ (quintet, $J=$ $7.2 \mathrm{~Hz}, 2 \mathrm{H}), 1.44$ (sextet, $J=7.2 \mathrm{~Hz}, 2 \mathrm{H}), 0.98(\mathrm{t}, J=7.2 \mathrm{~Hz}, 3 \mathrm{H}), 0.05(\mathrm{~s}, 9 \mathrm{H}) ;{ }^{13} \mathrm{C} \mathrm{NMR}$ $\left(\mathrm{CDCl}_{3}, 75 \mathrm{MHz}\right) \delta 170.9,133.8,131.1,128.7,127.5,98.0,74.6,48.5,29.5,19.7,13.7$, -0.2 ; HRMS (EI) calcd for $\mathrm{C}_{16} \mathrm{H}_{23} \mathrm{NOSi}[\mathrm{M}]^{+} 273.1549$, found 273.1552 .

$N$-Isopropyl- $N$-(trimethylsilanylethynyl)benzamide (2c). 


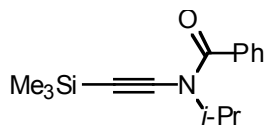

Yellow oil; IR (neat); 2925, 2150, 1660, 1290, 1240, 840, $690 \mathrm{~cm}^{-1} ;{ }^{1} \mathrm{H} \mathrm{NMR}\left(\mathrm{CDCl}_{3}\right.$, $300 \mathrm{MHz}) \delta 7.72-7.84(\mathrm{~m}, 2 \mathrm{H}), 7.31-7.51(\mathrm{~m}, 3 \mathrm{H}), 4.73$ (septet, $J=6.6 \mathrm{~Hz}, 1 \mathrm{H}), 1.30(\mathrm{~d}, J$ $=6.6 \mathrm{~Hz}, 6 \mathrm{H}), 0.06(\mathrm{~s}, 9 \mathrm{H}) ;{ }^{13} \mathrm{C} \mathrm{NMR}\left(\mathrm{CDCl}_{3}, 75 \mathrm{MHz}\right) \delta 170.7,134.2,131.0,128.7$, 127.5, 94.7, 77.1, 47.1, 19.9, -0.2; HRMS (EI) calcd for $\mathrm{C}_{15} \mathrm{H}_{21} \mathrm{NOSi}[\mathrm{M}]^{+} 259.1392$, found 259.1396.

\section{$N$-Phenyl- $N$-(trimethylsilanylethynyl)benzamide (2d).}

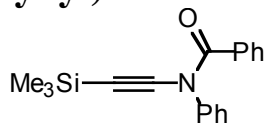

Yellow solid; Mp 70-71 ${ }^{\circ} \mathrm{C}$; IR (neat); 2975, 2200, 1680, 1260, 840, $720 \mathrm{~cm}^{-1}$; ${ }^{1} \mathrm{H}$ NMR $\left(\mathrm{CDCl}_{3}, 300 \mathrm{MHz}\right) \delta 7.78-7.89(\mathrm{~m}, 2 \mathrm{H}), 7.22-7.55(\mathrm{~m}, 8 \mathrm{H}), 0.06(\mathrm{~s}, 9 \mathrm{H}) ;{ }^{13} \mathrm{C} \mathrm{NMR}$ $\left(\mathrm{CDCl}_{3}, 75 \mathrm{MHz}\right) \delta 170.7,139.5,133.5,131.6,129.04,129.02,127.7,127.2,125.0,97.2$, 75.0, -0.3 ; HRMS (EI) calcd for $\mathrm{C}_{18} \mathrm{H}_{19} \mathrm{NOSi}\left[\mathrm{M}-\mathrm{CH}_{3}\right]^{+} 278.1001$, found 278.0956.

$N$-Benzyl- $N$-(trimethylsilanylethynyl)acetamide (2e).

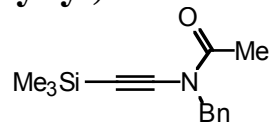

Yellow oil; IR (neat); 2900, 2150, 1690, 1360, 1240, 840, 740, $690 \mathrm{~cm}^{-1} ;{ }^{1} \mathrm{H}$ NMR $\left(\mathrm{CDCl}_{3}, 300 \mathrm{MHz}\right) \delta 7.27-7.39(\mathrm{~m}, 5 \mathrm{H}), 4.64(\mathrm{~s}, 2 \mathrm{H}), 2.33(\mathrm{~s}, 3 \mathrm{H}), 0.14(\mathrm{~s}, 9 \mathrm{H}) ;{ }^{13} \mathrm{C}$ NMR $\left(\mathrm{CDCl}_{3}, 75 \mathrm{MHz}\right) \delta 171.7,135.9,128.8,128.4,127.9,97.3,75.7,51.1,22.3,-0.03$; HRMS (EI) calcd for $\mathrm{C}_{14} \mathrm{H}_{19} \mathrm{NOSi}[\mathrm{M}]^{+} 245.1236$, found 245.1238 .

Benzyl(trimethylsilanylethynyl)carbamic acid methyl ester (2f).

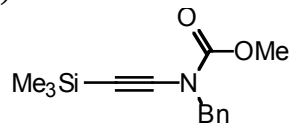

Yellow oil; IR (neat); 2900, 2175, 1720, 1460, 1360, 1250, 840, 760, $700 \mathrm{~cm}^{-1} ;{ }^{1} \mathrm{H}$ NMR $\left(\mathrm{CDCl}_{3}, 300 \mathrm{MHz}\right) \delta 7.22-7.41(\mathrm{~m}, 5 \mathrm{H}), 4.60(\mathrm{~s}, 2 \mathrm{H}), 3.81(\mathrm{~s}, 3 \mathrm{H}), 0.14(\mathrm{~s}, 9 \mathrm{H}) ;{ }^{13} \mathrm{C}$ NMR $\left(\mathrm{CDCl}_{3}, 75 \mathrm{MHz}\right) \delta 155.5,135.7,128.6,128.4,128.0,95.6,73.1,54.1,53.8,0.07$; HRMS (EI) calcd for $\mathrm{C}_{14} \mathrm{H}_{19} \mathrm{NO}_{2} \mathrm{Si}[\mathrm{M}-\mathrm{OMe}]^{+} 230.1001$, found 230.1059.

\section{Synthesis of 1,6-Diynes.}

\section{5,5-Bis(methoxymethyl)nona-2,7-diyne (1b). ${ }^{5}$

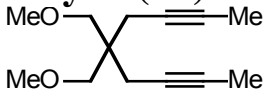

To a stirred mixture of $\mathrm{LiAlH}_{4}(182.3 \mathrm{mg}, 4.80 \mathrm{mmol})$ in $\mathrm{Et}_{2} \mathrm{O}(10 \mathrm{~mL})$ at $0{ }^{\circ} \mathrm{C}$ was added a solution of diyne $\mathbf{1 a}^{1}(497.2 \mathrm{mg}, 2.10 \mathrm{mmol})$ in $\mathrm{Et}_{2} \mathrm{O}(5 \mathrm{~mL})$. The resulting mixture was stirred at $0{ }^{\circ} \mathrm{C}$ for $30 \mathrm{~min}$. The reaction was quenched by the addition of water $(2.0$ $\mathrm{mL}$ ), followed by stirring at $0{ }^{\circ} \mathrm{C}$ for $10 \mathrm{~min}$. The solution was dried over $\mathrm{MgSO}_{4}$, filtered through Celite, and concentrated to give a diol (120.3 $\mathrm{mg}, 0.67 \mathrm{mmol}, 32 \%$ yield) as a colorless oil. To a stirred suspension of $\mathrm{NaH}$ (55\% in paraffin liquid, $147.8 \mathrm{mg}, 3.39 \mathrm{mmol}$ ) in THF $(5 \mathrm{ml})$ was added a THF $(5 \mathrm{~mL})$ solution of the diol $(218.4 \mathrm{mg}, 1.21 \mathrm{mmol})$ at $0{ }^{\circ} \mathrm{C}$, and the resulting mixture was stirred at $0{ }^{\circ} \mathrm{C}$ for $10 \mathrm{~min}$. MeI $(0.30 \mathrm{~mL}, 4.82 \mathrm{mmol})$ was 
added, and the resulting mixture was stirred at $\mathrm{rt}$ for $2 \mathrm{~h}$. The reaction mixture was extracted with ether. The organic layer was washed with saturated aqueous $\mathrm{NH}_{4} \mathrm{Cl}$ and brine, dried over $\mathrm{Na}_{2} \mathrm{SO}_{4}$, and concentrated. The crude product was purified by silica gel column chromatography (hexane:EtOAc $=20: 1$ ) afforded 5,5-bis-methoxymethylnona-2,7diyne (1b, $234.1 \mathrm{mg}, 1.12 \mathrm{mmol}, 93 \%$ yield) as a colorless oil. Colorless oil; ${ }^{1} \mathrm{H}$ NMR $\left(\mathrm{CDCl}_{3}, 300 \mathrm{MHz}\right) \delta 3.34(\mathrm{~s}, 6 \mathrm{H}), 3.32(\mathrm{~s}, 4 \mathrm{H}), 2.24(\mathrm{q}, J=2.4 \mathrm{~Hz}, 4 \mathrm{H}), 1.79(\mathrm{t}, J=2.4 \mathrm{~Hz}$, $6 \mathrm{H}) ;{ }^{13} \mathrm{C} \mathrm{NMR}\left(\mathrm{CDCl}_{3}, 75 \mathrm{MHz}\right) \delta 77.4,75.3,73.9,59.4,42.0,22.1,3.6$.

\section{Di(2-pentynyl) ether (1c). ${ }^{6}$}

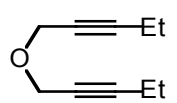

To a stirred suspension of $\mathrm{NaH}$ (55\% in paraffin liquid, $0.93 \mathrm{~g}, 21.3 \mathrm{mmol}$ ) in THF (20 $\mathrm{ml})$ was added a THF $(10 \mathrm{~mL})$ solution of 2-pentyn-1-ol $(0.80 \mathrm{~g}, 9.6 \mathrm{mmol})$ and 1-bromo2-pentyne $(1.68 \mathrm{~g}, 11.4 \mathrm{mmol})$ at $\mathrm{rt}$, and the resulting mixture was stirred at $\mathrm{rt}$ for $2 \mathrm{~h}$. The reaction mixture was extracted with ether. The organic layer was washed with brine, dried over $\mathrm{Na}_{2} \mathrm{SO}_{4}$, and concentrated. The crude product was purified by silica gel column chromatography (hexane:EtOAc = 50:1) afforded di(2-pentynyl) ether $(1 \mathrm{c}, 1.21 \mathrm{~g}, 8.04$ mmol, $84 \%$ yield) as a colorless oil. Colorless oil; ${ }^{1} \mathrm{H}$ NMR $\left(\mathrm{CDCl}_{3}, 300 \mathrm{MHz}\right) \delta 4.21(\mathrm{t}, J$ $=2.4 \mathrm{~Hz}, 4 \mathrm{H}), 2.23(\mathrm{tq}, J=7.5$ and $2.4 \mathrm{~Hz}, 4 \mathrm{H}), 1.15(\mathrm{t}, J=7.5 \mathrm{~Hz}, 6 \mathrm{H}) ;{ }^{13} \mathrm{C} \mathrm{NMR}\left(\mathrm{CDCl}_{3}\right.$, $75 \mathrm{MHz}) \delta 88.6,74.6,56.9,13.7,12.4$.

\section{4-Bromo- $N, N$-dibut-2-ynylbenzenesulfonamide (1d).}

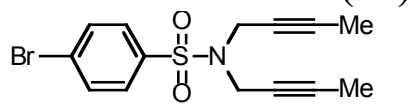

To a stirred suspension of $\mathrm{K}_{2} \mathrm{CO}_{3}(1.74 \mathrm{~g}, 12.6 \mathrm{mmol})$ in $\mathrm{CH}_{3} \mathrm{CN}$ (15 ml) was added 4bromobenzenesulfoamide $(0.60 \mathrm{~g}, 2.52 \mathrm{mmol})$ and 1-bromo-2-butyne $(0.55 \mathrm{~mL}, 6.28$ $\mathrm{mmol}$ ), and the resulting mixture was refluxed for $2 \mathrm{~h}$. The reaction mixture was extracted with ether. The organic layer was washed with brine, dried over $\mathrm{Na}_{2} \mathrm{SO}_{4}$, and concentrated. The crude product was purified by silica gel column chromatography (hexane:EtOAc $=$ 30:1) afforded 4-bromo- $N, N$-dibut-2-ynylbenzenesulfonamide (1d, 0.80 g, 2.35 mmol, 93\% yield) as a pale yellow oil.

Pale yellow oil; IR (neat); 3300, 2900, 1570, 1425, 1330, 1150, 1090, 1060, 900, 750 $\mathrm{cm}^{-1} ;{ }^{1} \mathrm{H}$ NMR $\left(\mathrm{CDCl}_{3}, 300 \mathrm{MHz}\right) \delta 7.60-7.74(\mathrm{~m}, 4 \mathrm{H}), 4.08(\mathrm{q}, J=2.4 \mathrm{~Hz}, 4 \mathrm{H}), 1.66(\mathrm{t}, J$ $=2.4 \mathrm{~Hz}, 6 \mathrm{H}) ;{ }^{13} \mathrm{C} \mathrm{NMR}\left(\mathrm{CDCl}_{3}, 75 \mathrm{MHz}\right) \delta 137.5,131.8,129.5,127.7,82.0,71.3,36.7$, 3.4; HRMS (EI) calcd for $\mathrm{C}_{14} \mathrm{H}_{14} \mathrm{BrNO}_{2} \mathrm{~S}\left[\mathrm{M}-\mathrm{CH}_{3}\right]^{+}$323.9694, found 323.9667 .

\section{Rhodium-Catalyzed Enantioselective [2+2+2] Cycloaddition of 1,6-Diynes with Trimethylsilylynamides (Table 2 and eq 1)}

General Procedure (Table 2, entry 1). Under an Ar atmosphere, $(S)$-xyl-BINAP (18.4 $\mathrm{mg}, 0.0250 \mathrm{mmol})$ and $\left[\mathrm{Rh}(\mathrm{cod})_{2}\right] \mathrm{BF}_{4}(10.2 \mathrm{mg}, 0.0250 \mathrm{mmol})$ were dissolved in $\mathrm{CH}_{2} \mathrm{Cl}_{2}$ $(1.0 \mathrm{~mL})$ and the mixture was stirred at $\mathrm{rt}$ for $5 \mathrm{~min} . \mathrm{H}_{2}$ was introduced to the resulting solution in a Schlenk tube. After stirring at $\mathrm{rt}$ for $1 \mathrm{~h}$, the resulting solution was concentrated to dryness and dissolved in $\mathrm{CH}_{2} \mathrm{Cl}_{2}(1.0 \mathrm{~mL})$. To this solution was added dropwise over $1 \mathrm{~min}$ a solution of diyne $1 \mathbf{a}(59.1 \mathrm{mg}, 0.250 \mathrm{mmol})$ and 
trimethylsilylynamide $2 \mathbf{a}(76.9 \mathrm{mg}, 0.250 \mathrm{mmol})$ in $\mathrm{CH}_{2} \mathrm{Cl}_{2}(1.0 \mathrm{~mL})$ at $\mathrm{rt}$ and washed remaining substrates away by using $\mathrm{CH}_{2} \mathrm{Cl}_{2}(3.0 \mathrm{~mL})$. The mixture was stirred at $\mathrm{rt}$ for $38 \mathrm{~h}$. The resulting solution was concentrated and purified by preparative TLC (hexane:EtOAc: $\mathrm{Et}_{3} \mathrm{~N}=10: 1: 2$ ), which furnished 5-(benzoylbenzylamino)-4,7-dimethyl-6(trimethylsilanyl)indan-2,2-dicarboxylic acid dimethyl ester (3aa, 38.9 mg, $0.072 \mathrm{mmol}$, $29 \%$ yield) as a colorless oil.

(+)-5-(Benzoylbenzylamino)-4,7-dimethyl-6-(trimethylsilanyl)indan-2,2-dicarboxylic acid dimethyl ester (3aa, entry 1).

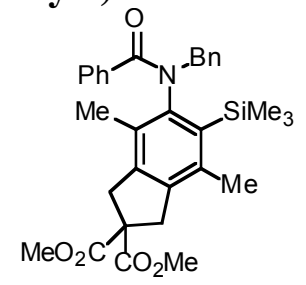

Colorless oil; $[\alpha]_{D}^{25}+101.0^{\circ}\left(c 0.845, \mathrm{CHCl}_{3}, 97 \%\right.$ ee); IR (neat); 2900, 1720, 1620, 1380, 1250, 1180, 840, $700 \mathrm{~cm}^{-1}$; ${ }^{1} \mathrm{H}$ NMR $\left(\mathrm{CDCl}_{3}, 300 \mathrm{MHz}\right) \delta 7.36-7.44(\mathrm{~m}, 2 \mathrm{H})$, 7.03-7.28 (m, 8H), $5.93(\mathrm{~d}, J=12.9 \mathrm{~Hz}, 1 \mathrm{H}), 3.85(\mathrm{~d}, J=12.9 \mathrm{~Hz}, 1 \mathrm{H}), 3.76(\mathrm{~s}, 3 \mathrm{H}), 3.69$ (s, 3H), $3.52(\mathrm{~s}, 2 \mathrm{H}), 3.20(\mathrm{~d}, J=17.1 \mathrm{~Hz}, 1 \mathrm{H}), 3.07(\mathrm{~d}, J=17.1 \mathrm{~Hz}, 1 \mathrm{H}), 2.43(\mathrm{~s}, 3 \mathrm{H}), 0.82$ $(\mathrm{s}, 3 \mathrm{H}), 0.50(\mathrm{~s}, 9 \mathrm{H}) ;{ }^{13} \mathrm{C} \mathrm{NMR}\left(\mathrm{CDCl}_{3}, 75 \mathrm{MHz}\right) \delta 172.2,172.0,168.3,145.2,141.1$, 138.6, 135.7, 135.5, 134.7, 130.6, 130.4, 129.7, 129.4, 127.9, 127.7, 127.3, 58.9, 57.1, 53.0, 52.9, 40.4, 40.1, 20.5, 14.3, 2.4; HRMS (ESI) calcd for $\mathrm{C}_{32} \mathrm{H}_{37} \mathrm{NO}_{5} \mathrm{Si}[\mathrm{M}+\mathrm{Na}]^{+}$566.2339, found 566.2350. CHIRALPAK AD, hexane:2-PrOH =95:5, $1.0 \mathrm{~mL} / \mathrm{min}$, retention times: $21.0 \mathrm{~min}$ (major isomer) and $26.3 \mathrm{~min}$ (minor isomer).

(+)-5-(Benzoylbutylamino)-4,7-dimethyl-6-(trimethylsilanyl)indan-2,2-dicarboxylic acid dimethyl ester (3ab, entry 2, reaction time: $18 \mathrm{~h}$ ).

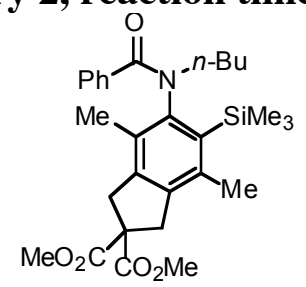

Pale yellow oil; $[\alpha]^{25}+125.6^{\circ}\left(c 0.800, \mathrm{CHCl}_{3}, 97 \%\right.$ ee); IR (neat); 2900, 1730, 1620, 1400, 1240, 840, $700 \mathrm{~cm}^{-1} ;{ }^{1} \mathrm{H}$ NMR $\left(\mathrm{CDCl}_{3}, 300 \mathrm{MHz}\right) \delta 7.29-7.40(\mathrm{~m}, 2 \mathrm{H}), 7.17-7.29$ (m, 1H), 7.05-7.17 (m, 2H), $4.44(\mathrm{dt}, J=12.3$ and $4.5 \mathrm{~Hz}, 1 \mathrm{H}), 3.76(\mathrm{~s}, 3 \mathrm{H}), 3.73(\mathrm{~s}, 3 \mathrm{H})$, $3.53(\mathrm{~s}, 2 \mathrm{H}), 3.44(\mathrm{~d}, J=16.8 \mathrm{~Hz}, 1 \mathrm{H}), 3.28(\mathrm{~d}, J=16.8 \mathrm{~Hz}, 1 \mathrm{H}), 2.99(\mathrm{dt}, J=12.3$ and 4.5 $\mathrm{Hz}, 1 \mathrm{H}), 2.36(\mathrm{~s}, 3 \mathrm{H}), 1.73(\mathrm{~s}, 3 \mathrm{H}), 1.57-1.76(\mathrm{~m}, 1 \mathrm{H}), 1.13-1.43(\mathrm{~m}, 3 \mathrm{H}), 0.89$ (t, $J=7.2$ $\mathrm{Hz}, 3 \mathrm{H}), 0.40(\mathrm{~s}, 9 \mathrm{H}) ;{ }^{13} \mathrm{C} \mathrm{NMR}\left(\mathrm{CDCl}_{3}, 75 \mathrm{MHz}\right) \delta 172.1,168.4,145.7,141.1,138.6$, $138.5,135.9$, 135.3, 129.6, 129.23, 129.16, 127.3, 58.8, 53.9, 53.1, 53.0, 40.5, 40.3, 28.0, 20.6, 20.4, 15.6, 13.8, 2.2; HRMS (ESI) calcd for $\mathrm{C}_{29} \mathrm{H}_{39} \mathrm{NO}_{5} \mathrm{Si}[\mathrm{M}+\mathrm{Na}]^{+} 532.2495$, found 532.2511. CHIRALPAK AD-H, hexane:2-PrOH = 90:10, $1.0 \mathrm{~mL} / \mathrm{min}$, retention times: 15.2 min (minor isomer) and $17.4 \mathrm{~min}$ (major isomer).

(-)-5-(Benzoylisopropylamino)-4,7-dimethyl-6-(trimethylsilanyl)indan-2,2-dicarboxylic acid dimethyl ester (3ac, entry 3 , reaction time: $15 \mathrm{~h}$ ). 


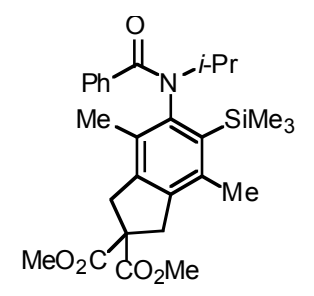

Colorless oil; $[\alpha]_{\mathrm{D}}^{25}-1.20^{\circ}$ (c 1.255, $\mathrm{CHCl}_{3}, 87 \%$ ee); IR (neat); 2900, 1720, 1620, 1340, 1240, 1160, 840, $700 \mathrm{~cm}^{-1}$; ${ }^{1} \mathrm{H}$ NMR $\left(\mathrm{CDCl}_{3}, 300 \mathrm{MHz}\right) \delta 7.13-7.25(\mathrm{~m}, 3 \mathrm{H})$, 7.01-7.13 (m, 2H), 4.67 (septet, $J=6.6 \mathrm{~Hz}, 1 \mathrm{H}), 3.78(\mathrm{~s}, 3 \mathrm{H}), 3.77(\mathrm{~s}, 3 \mathrm{H}), 3.53(\mathrm{~s}, 2 \mathrm{H})$, $3.52(\mathrm{~d}, J=16.8 \mathrm{~Hz}, 1 \mathrm{H}), 3.44(\mathrm{~d}, J=16.8 \mathrm{~Hz}, 1 \mathrm{H}), 2.27(\mathrm{~s}, 3 \mathrm{H}), 2.06(\mathrm{~s}, 3 \mathrm{H}), 1.45(\mathrm{~d}, J=$ $6.6 \mathrm{~Hz}, 3 \mathrm{H}), 0.84(\mathrm{~d}, J=6.6 \mathrm{~Hz}, 3 \mathrm{H}), 0.25(\mathrm{~s}, 9 \mathrm{H}) ;{ }^{13} \mathrm{C} \mathrm{NMR}\left(\mathrm{CDCl}_{3}, 75 \mathrm{MHz}\right) \delta 172.20$, 172.16, 168.6, 142.6, 141.1, 139.0, 138.7, 137.4, 136.7, 130.5, 129.2, 128.9, 127.2, 58.8, 53.1, 53.0, 51.0, 40.5, 40.4, 22.1, 20.6, 20.3, 17.2, 2.3; HRMS (ESI) calcd for $\mathrm{C}_{28} \mathrm{H}_{37} \mathrm{NO}_{5} \mathrm{Si}$ $[\mathrm{M}+\mathrm{Na}]^{+}$518.2339, found 518.2346. CHIRALPAK AD-H, hexane:2-PrOH = 90:10, 1.0 $\mathrm{mL} / \mathrm{min}$, retention times: $7.8 \mathrm{~min}$ (minor isomer) and $14.7 \mathrm{~min}$ (major isomer).

(-)-5-(Benzoylphenylamino)-4,7-dimethyl-6-(trimethylsilanyl)indan-2,2-dicarboxylic acid dimethyl ester (3ad, entry 4, cis:trans $=60: 40$, reaction time: $42 \mathrm{~h}$ ).<smiles>CC(C)=O</smiles>

The ratio of cis:trans was determined by ${ }^{1} \mathrm{H}$ NMR chemical shift of Me singlet adjacent to amide group. The hghfield Me singlet of the anilide was assigned to the cis-rotamer. Colorless oil; $[\alpha]^{25}-32.6^{\circ}$ (c 1.140, $\mathrm{CHCl}_{3}, 97 \%$ ee); IR (neat); 2900, 1720, 1640, 1430, $1230,840,710 \mathrm{~cm}^{-1} ;{ }^{1} \mathrm{H}$ NMR $\left(\mathrm{CDCl}_{3}, 300 \mathrm{MHz}\right) \delta 6.91-7.58(\mathrm{~m}, 10 \mathrm{H}$, cis; $8 \mathrm{H}$, trans), 6.56-6.68 (m, $2 \mathrm{H}$, trans $), 3.70-3.84(\mathrm{~m}, 6 \mathrm{H}$, cis and trans $), 3.38-3.70(\mathrm{~m}, 4 \mathrm{H}$, cis and trans), 2.32 (s, 3H, trans), 2.25 (s, 3H, cis), 2.18 (s, 3H, trans), $1.95(\mathrm{~s}, 3 \mathrm{H}$, cis $), 0.22(\mathrm{~s}$, $9 \mathrm{H}$, trans $), 0.16(\mathrm{~s}, 9 \mathrm{H}$, cis $) ;{ }^{13} \mathrm{C}$ NMR $\left(\mathrm{CDCl}_{3}, 75 \mathrm{MHz}\right) \delta 172.6,172.1,172.0,169.8$, 169.0, 146.3, 145.3, 143.1, 142.9, 141.6, 141.5, 139.5, 139.0, 138.8, 138.2, 137.7, 136.6, 136.1, 135.6, 130.3, 130.0, 129.8, 129.3, 129.0, 128.2, 128.0, 127.9, 127.4, 125.0, 124.4, 124.3, 123.9, 58.9, 53.1, 40.7, 40.5, 40.2, 21.0, 20.3, 15.3, 15.1, 2.2; HRMS (ESI) calcd for $\mathrm{C}_{31} \mathrm{H}_{35} \mathrm{NO}_{5} \mathrm{Si}[\mathrm{M}+\mathrm{Na}]^{+}$552.2182, found 552.2241. CHIRALPAK AD, hexane:2-PrOH = 80:20,1.0 $\mathrm{mL} / \mathrm{min}$, retention times: $11.8 \mathrm{~min}$ (minor isomer) and $23.9 \mathrm{~min}$ (major isomer).

(+)-5-(Acetylbenzylamino)-4,7-dimethyl-6-(trimethylsilanyl)indan-2,2-dicarboxylic acid dimethyl ester (3ae, entry 5 , reaction time: $16 \mathrm{~h}$ ).

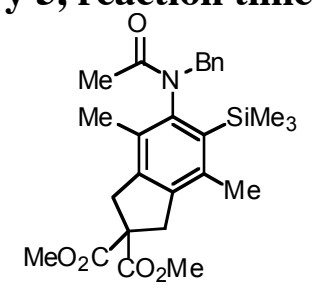

Colorless oil; $[\alpha]_{\mathrm{D}}^{25}+35.9^{\circ}\left(c\right.$ 1.235, $\mathrm{CHCl}_{3}, 90 \%$ ee); IR (neat); 2900, 1720, 1640, 
1390, 1240, 1180, 840, $700 \mathrm{~cm}^{-1}$; ${ }^{1} \mathrm{H}$ NMR $\left(\mathrm{CDCl}_{3}, 300 \mathrm{MHz}\right) \delta 7.11-7.31(\mathrm{~m}, 3 \mathrm{H})$, 7.00-7.11 (m, 2H), $5.66(\mathrm{~d}, J=12.9 \mathrm{~Hz}, 1 \mathrm{H}), 3.80(\mathrm{~s}, 3 \mathrm{H}), 3.76(\mathrm{~s}, 3 \mathrm{H}), 3.66(\mathrm{~d}, J=12.9$ $\mathrm{Hz}, 1 \mathrm{H}), 3.56(\mathrm{~s}, 2 \mathrm{H}), 3.36(\mathrm{~s}, 2 \mathrm{H}), 2.38(\mathrm{~s}, 3 \mathrm{H}), 1.79(\mathrm{~s}, 3 \mathrm{H}), 1.19(\mathrm{~s}, 3 \mathrm{H}), 0.36(\mathrm{~s}, 9 \mathrm{H}) ;{ }^{13} \mathrm{C}$ NMR $\left(\mathrm{CDCl}_{3}, 75 \mathrm{MHz}\right) \delta 172.2,170.5,144.8,141.3,138.9,138.7,135.9,134.7,130.5$, $130.4,128.1,127.6,58.9,54.7,53.1,40.5,40.2$, 22.8, 20.5, 14.2, 2.3; HRMS (ESI) calcd for $\mathrm{C}_{27} \mathrm{H}_{35} \mathrm{NO}_{5} \mathrm{Si}[\mathrm{M}+\mathrm{Na}]^{+}$504.2182, found 504.2200. CHIRALPAK AD-H, hexane:2$\mathrm{PrOH}=96: 4,1.0 \mathrm{~mL} / \mathrm{min}$, retention times: $13.3 \mathrm{~min}$ (major isomer) and $15.2 \mathrm{~min}$ (minor isomer).

(-)-Benzyl[2-(4-bromobenzenesulfonyl)-4,7-dimethyl-6-(trimethylsilanyl)-2,3dihydro-1H-isoindol-5-yl]carbamic acid methyl ester (3df, entry 6, cis:trans = 86:14, reaction time: $15 \mathrm{~h}$ ).

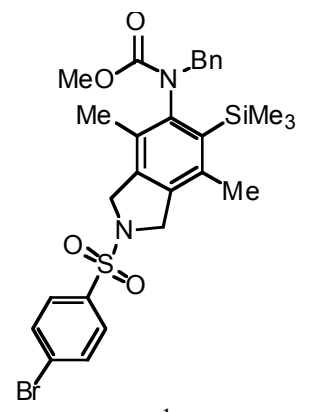

The ratio of cis:trans was determined by ${ }^{1} \mathrm{H}$ NMR chemical shift of $\mathrm{MeO}$ singlet. The hghfield $\mathrm{MeO}$ singlet of the anilide was assigned to the $c i s$-rotamer. Pale yellow oil; $[\alpha]_{\mathrm{D}}^{25}$ $-20.3^{\circ}$ (c 5.810, $\mathrm{CHCl}_{3}, 98 \%$ ee); IR (neat); 2900, 1680, 1570, 1320, 1150, 1100, 1060, 820, 730, $690 \mathrm{~cm}^{-1} ;{ }^{1} \mathrm{H} \mathrm{NMR}\left(\mathrm{CDCl}_{3}, 300 \mathrm{MHz}\right) \delta 7.64-7.78,7.13-7.30$, and 6.93-7.06 (m, $9 \mathrm{H}$, cis and trans), $5.27(\mathrm{~d}, J=13.8 \mathrm{~Hz}, 1 \mathrm{H}$, cis $), 5.14(\mathrm{~d}, J=13.8 \mathrm{~Hz}, 1 \mathrm{H}$, trans), 4.40-4.52 (m, 2H, cis and trans), $4.32(\mathrm{dd}, J=12.3$ and $3.0 \mathrm{~Hz}, 1 \mathrm{H}$, cis and trans), $3.92(\mathrm{~d}$, $J=13.8 \mathrm{~Hz}, 1 \mathrm{H}$, trans), $3.86(\mathrm{~d}, J=13.8 \mathrm{~Hz}, 1 \mathrm{H}$, cis $), 3.85(\mathrm{dd}, J=12.3$ and $3.0 \mathrm{~Hz}, 1 \mathrm{H}$, cis and trans), $3.85(\mathrm{~s}, 3 \mathrm{H}$, trans), $3.63(\mathrm{~s}, 3 \mathrm{H}$, cis), 2.31 (s, 3H, cis), 2.29 (s, 3H, trans), 1.21 (s, 3H, trans), 1.18 (s, 3H, cis), 0.34 (s, 9H, trans), 0.33 (s, 9H, cis) ${ }^{13} \mathrm{C} \mathrm{NMR}\left(\mathrm{CDCl}_{3}\right.$, $75 \mathrm{MHz}) \delta 155.9,144.4,137.3,136.8,136.5,135.8,135.7,134.6,132.5,130.0,129.6$, 128.9, 128.2, 127.94, 127.88, 56.5, 54.0, 53.7, 53.0, 20.1, 13.9, 1.9; HRMS (ESI) calcd for $\mathrm{C}_{28} \mathrm{H}_{33} \mathrm{BrN}_{2} \mathrm{O}_{4} \mathrm{SSi}[\mathrm{M}+\mathrm{Na}]^{+}$623.1011, found 623.1044. CHIRALPAK AD, hexane:2-PrOH $=85: 15,1.0 \mathrm{~mL} / \mathrm{min}$, retention times: $8.6 \mathrm{~min}$ (minor isomer) and $17.2 \mathrm{~min}$ (major isomer).

(-)- $N$-[2,2-Dimethoxymethyl-4,7-dimethyl-6-(trimethylsilanyl)indan-5-yl]- $N$ phenylbenzamide (3bd, entry 7, cis:trans $=59: 41$, reaction time: $16 \mathrm{~h}$ ).

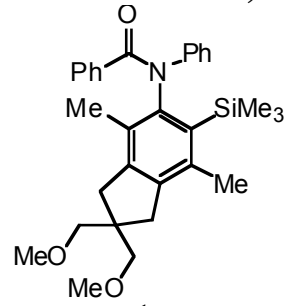

The ratio of cis:trans was determined by ${ }^{1} \mathrm{H}$ NMR chemical shift of Me singlet adjacent to amide group. The hghfield Me singlet of the anilide was assigned to the cis-rotamer. Pale yellow oil; $[\alpha]_{D}^{25}-17.5^{\circ}$ (c 2.065, $\mathrm{CHCl}_{3}, 98 \%$ ee); IR (neat); 2850, 1640, 1300, 1100, 840, 
740, $690 \mathrm{~cm}^{-1} ;{ }^{1} \mathrm{H}$ NMR $\left(\mathrm{CDCl}_{3}, 300 \mathrm{MHz}\right) \delta 6.90-7.55(\mathrm{~m}, 10 \mathrm{H}$, cis; 8H, trans), 6.56-6.70 (m, 2H, trans), 3.21-3.51 (m, 10H, cis and trans), 2.62-2.94 (m, 4H, cis and trans), $2.28(\mathrm{~s}$, 3H, trans), 2.21 (s, 3H, cis), 2.15 (s, 3H, trans), 1.91 (s, 3H, cis), 0.22 (s, 9H, trans), 0.16 $(\mathrm{s}, 9 \mathrm{H}, \mathrm{cis}) ;{ }^{13} \mathrm{C} \mathrm{NMR}\left(\mathrm{CDCl}_{3}, 75 \mathrm{MHz}\right) \delta 169.9,169.1,145.7,144.6,143.8,143.6,143.2$, $143.1,141.7,141.1,139.4,138.6,136.9$, 136.6, 136.3, 134.5, 132.2, 130.4, 130.1, 129.7, $129.6,128.9$, 128.1 , 128.0, 127.9, 127.2, 125.0, 124.24, 124.19, 123.9, 76.3, 76.24, 76.16, 59.3, 59.2, 46.8, 46.5, 39.0, 38.7, 38.5, 21.0, 20.3, 15.3, 15.0, 2.2; HRMS (ESI) calcd for $\mathrm{C}_{31} \mathrm{H}_{39} \mathrm{NO}_{3} \mathrm{Si}[\mathrm{M}+\mathrm{Na}]^{+}$524.2597, found 524.2623. CHIRALPAK AD-H, hexane:2-PrOH = 80:20, $1.0 \mathrm{~mL} / \mathrm{min}$, retention times: $6.0 \mathrm{~min}$ (minor isomer) and $7.6 \mathrm{~min}$ (major isomer).

(-)- $N$-[4,7-Diethyl-6-(trimethylsilanyl)-1,3-dihydroisobenzofuran-5-yl]- $N$-phenylbenzamide (3cd, entry 8, cis:trans $=73: 27$, reaction time: 16 h).<smiles>CCc1c2c(c(CC)c(N(C(=O)C(=O)O)c3ccccc3)c1CC)COC2</smiles>

The ratio of cis:trans was determined by ${ }^{1} \mathrm{H}$ NMR chemical shift of Et triplet adjacent to amide group. The hghfield Et triplet of the anilide was assigned to the cis-rotamer. Yellow oil; $[\alpha]_{D}^{25}-178.2^{\circ}$ (c 3.495, $\mathrm{CHCl}_{3}, 96 \%$ ee); IR (neat); 2850, 1640, 1580, 1280, 1050, $840,740,690 \mathrm{~cm}^{-1} ;{ }^{1} \mathrm{H}$ NMR $\left(\mathrm{CDCl}_{3}, 300 \mathrm{MHz}\right) \delta 6.93-7.57(\mathrm{~m}, 10 \mathrm{H}$, cis; 8H, trans), 6.62-6.70 (m, 2H, trans), $5.20(\mathrm{~s}, 2 \mathrm{H}$, trans $), 5.17(\mathrm{~s}, 2 \mathrm{H}$, trans $), 5.13(\mathrm{~s}, 2 \mathrm{H}$, cis $)$, 5.10 (s, 2H, cis), 2.24-2.88 (m, 4H, cis and trans), $1.15(\mathrm{t}, J=7.5 \mathrm{~Hz}, 3 \mathrm{H}$, trans $), 0.94(\mathrm{t}, J$ $=7.5 \mathrm{~Hz}, 3 \mathrm{H}$, trans $), 0.84(\mathrm{t}, J=7.5 \mathrm{~Hz}, 3 \mathrm{H}$, cis $), 0.49(\mathrm{t}, J=7.5 \mathrm{~Hz}, 3 \mathrm{H}$, cis $), 0.27(\mathrm{~s}, 9 \mathrm{H}$, trans), $0.15(\mathrm{~s}, 9 \mathrm{H}$, cis $) ;{ }^{13} \mathrm{C} \mathrm{NMR}\left(\mathrm{CDCl}_{3}, 75 \mathrm{MHz}\right) \delta 170.4,168.8,145.8,145.3,144.0$, $143.44,143.38,143.0,141.7,141.5,138.61,138.57,138.4,136.4,136.0,133.0,130.4$, $129.7,129.1,129.0,128.3,128.0,127.9,127.3,125.0,124.7,124.5,124.2,73.4,73.1,72.8$, 27.2, 26.8, 23.2, 23.0, 14.9, 14.8, 12.7, 11.7, 3.0, 2.8; HRMS (ESI) calcd for $\mathrm{C}_{28} \mathrm{H}_{33} \mathrm{NO}_{2} \mathrm{Si}$ $[\mathrm{M}+\mathrm{Na}]^{+}$466.2178, found 466.2200. CHIRALPAK AD-H, hexane:2-PrOH $=70: 30,1.0$ $\mathrm{mL} / \mathrm{min}$, retention times: $7.5 \mathrm{~min}$ (minor isomer) and $20.5 \mathrm{~min}$ (major isomer).

(+)- $N$-[2-(4-Bromobenzenesulfonyl)-4,7-dimethyl-6-(trimethylsilanyl)-2,3-dihydro-1H-isoindol-5-yl]- $N$-phenylbenzamide (3dd, entry 9, cis:trans $=45: 55$, reaction time: $40 \mathrm{~h}$ ).

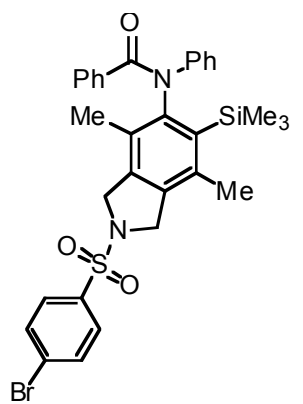

The ratio of cis:trans was determined by ${ }^{1} \mathrm{H}$ NMR chemical shift of Me singlet adjacent to amide group. The hghfield Me singlet of the anilide was assigned to the cis-rotamer. Yellow oil; $[\alpha]_{\mathrm{D}}^{25}+5.07^{\circ}$ (c 5.790, $\mathrm{CHCl}_{3}, 84 \%$ ee); IR (neat); 2900, 1640, 1570, 1300, 
1160, 830, 730, $690 \mathrm{~cm}^{-1} ;{ }^{1} \mathrm{H}$ NMR $\left(\mathrm{CDCl}_{3}, 300 \mathrm{MHz}\right) \delta 7.62-7.87(\mathrm{~m}, 4 \mathrm{H}$, cis and trans), 6.93-7.50 (m, 10H, cis; 8H, trans), 6.53-6.68 (m, 2H, trans), 4.32-4.84 (m, 4H, cis and trans), 2.27 (s, 3H, trans), 2.19 (s, 3H, cis), 2.10 (s, 3H, trans), 1.89 (s, 3H, cis), 0.22 (s, 9H, trans), 0.17 (s, 9H, cis); ${ }^{13} \mathrm{C} \mathrm{NMR}\left(\mathrm{CDCl}_{3}, 75 \mathrm{MHz}\right) \delta 169.8,168.8,147.0,146.1$, $142.8,142.4$, 139.5, 137.8, 137.6, 137.5, 137.4, 137.2, 136.2, 135.9, 135.7, 135.6, 135.5, $135.1,132.5,132.4,131.7,130.5,130.0,129.7,128.93,128.85,128.8,128.3,128.2$, $128.04,127.97,127.9,127.8,127.5,124.8,124.6,123.8,54.2,54.1,53.9,53.6,20.5,19.9$, 15.2, 15.0, 2.0; HRMS (ESI) calcd for $\mathrm{C}_{32} \mathrm{H}_{33} \mathrm{BrN}_{2} \mathrm{O}_{3} \mathrm{SSi}[\mathrm{M}+\mathrm{Na}]^{+}$655.1062, found 655.1105. CHIRALPAK AD-H, hexane:2-PrOH =60:40, $0.9 \mathrm{~mL} / \mathrm{min}$, retention times: 42.1 min (minor isomer) and 74.8 min (major isomer).

(S)-(+)- $N$-Benzyl- $N$-[4,7-dimethyl-2-(toluene-4-sulfonyl)-6-(trimethylsilanyl)-2,3dihydro-1H-isoindol-5-yl]benzamide (3ea, entry 10, reaction time: $15 \mathrm{~h}$ ).

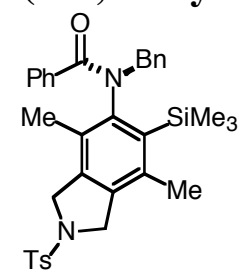

Colorless solid; $\mathrm{Mp} 210-211^{\circ} \mathrm{C} ;[\alpha]_{\mathrm{D}}^{25}+87.6^{\circ}$ (c 0.920, $\mathrm{CHCl}_{3}, 79 \%$ ee); IR (neat); 2900, 1620, 1380, 1330, 1250, 1160, 1100, 840, 700, $660 \mathrm{~cm}^{-1} ;{ }^{1} \mathrm{H} \mathrm{NMR}\left(\mathrm{CDCl}_{3}, 300\right.$ $\mathrm{MHz}) \delta 7.69-7.79(\mathrm{~m}, 2 \mathrm{H}), 7.02-7.41(\mathrm{~m}, 12 \mathrm{H}), 5.93(\mathrm{~d}, J=12.9 \mathrm{~Hz}, 1 \mathrm{H}), 4.58(\mathrm{~d}, J=$ $13.5 \mathrm{~Hz}, 1 \mathrm{H}), 4.51(\mathrm{~d}, J=13.5 \mathrm{~Hz}, 1 \mathrm{H}), 4.21(\mathrm{~d}, J=13.5 \mathrm{~Hz}, 1 \mathrm{H}), 4.09(\mathrm{~d}, J=13.5 \mathrm{~Hz}$, $1 \mathrm{H}), 3.79(\mathrm{~d}, J=12.9 \mathrm{~Hz}, 1 \mathrm{H}), 2.43(\mathrm{~s}, 3 \mathrm{H}), 2.37(\mathrm{~s}, 3 \mathrm{H}), 0.77(\mathrm{~s}, 3 \mathrm{H}), 0.51(\mathrm{~s}, 9 \mathrm{H}) ;{ }^{13} \mathrm{C}$ NMR $\left(\mathrm{CDCl}_{3}, 75 \mathrm{MHz}\right) \delta 168.3,145.9,143.6,137.6,137.4,136.4,135.5,135.3,135.1$, 134.2, 130.4, 129.9, 129.8, 129.3, 129.2, 128.1, 127.9, 127.5, 127.4, 57.1, 53.8, 53.4, 21.5, 20.1, 14.1, 2.2; HRMS (ESI) calcd for $\mathrm{C}_{34} \mathrm{H}_{38} \mathrm{~N}_{2} \mathrm{O}_{3} \mathrm{SSi}[\mathrm{M}+\mathrm{Na}]^{+}$605.2270, found 605.2289. CHIRALPAK AD, hexane:2-PrOH = 75:25, $1.0 \mathrm{~mL} / \mathrm{min}$, retention times: $29.7 \mathrm{~min}$ (major isomer) and $34.6 \mathrm{~min}$ (minor isomer). The absolute configuration of (+)-3ea was determined to be $S$ by anomalous dispersion method. The single crystals which are suitable for X-ray crystallographic analysis were obtained by recrystallization from acetone/pentane.

(+)- $N$-Benzyl- $N$-[4,7-dimethyl-2-(toluene-4-sulfonyl)-6-(trimethylsilanyl)-2,3dihydro-1H-isoindol-5-yl]acetamide (3ee, entry 11, reaction time: $16 \mathrm{~h}$ ).

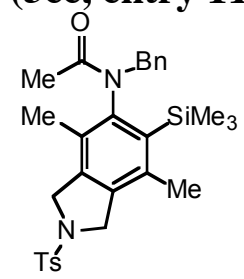

Pale yellow oil; $[\alpha]_{\mathrm{D}}^{25}+47.6^{\circ}$ (c 1.950, $\mathrm{CHCl}_{3}, 88 \%$ ee); IR (neat); 2900, 1630, 1390, 1330, 1160, 1100, 830, 740, $660 \mathrm{~cm}^{-1} ;{ }^{1} \mathrm{H} \mathrm{NMR}\left(\mathrm{CDCl}_{3}, 300 \mathrm{MHz}\right) \delta 7.72-7.87(\mathrm{~m}, 2 \mathrm{H})$, 6.97-7.41 (m, 7H), $5.67(\mathrm{~d}, J=13.2 \mathrm{~Hz}, 1 \mathrm{H}), 4.64(\mathrm{~d}, J=13.5 \mathrm{~Hz}, 1 \mathrm{H}), 4.52(\mathrm{~d}, J=13.5$ $\mathrm{Hz}, 1 \mathrm{H}), 4.42(\mathrm{~d}, J=13.8 \mathrm{~Hz}, 1 \mathrm{H}), 4.33(\mathrm{~d}, J=13.8 \mathrm{~Hz}, 1 \mathrm{H}), 3.61(\mathrm{~d}, J=13.2 \mathrm{~Hz}, 1 \mathrm{H})$, $2.43(\mathrm{~s}, 3 \mathrm{H}), 2.32(\mathrm{~s}, 3 \mathrm{H}), 1.74(\mathrm{~s}, 3 \mathrm{H}), 1.13(\mathrm{~s}, 3 \mathrm{H}), 0.36(\mathrm{~s}, 9 \mathrm{H}) ;{ }^{13} \mathrm{C} \mathrm{NMR}\left(\mathrm{CDCl}_{3}, 75\right.$ 
MHz) $\delta 170.1,145.4,143.7,137.7,137.6,136.4,135.7,135.5,133.9,130.4,129.8,129.4$, 128.2, 127.8, 127.5, 54.8, 53.8, 53.5, 22.7, 21.5, 20.0, 13.9, 2.1; HRMS (ESI) calcd for $\mathrm{C}_{29} \mathrm{H}_{36} \mathrm{~N}_{2} \mathrm{O}_{3} \mathrm{SSi}[\mathrm{M}+\mathrm{Na}]^{+}$543.2114, found 543.2126. CHIRALPAK AD, hexane:2-PrOH = 75:25, $1.0 \mathrm{~mL} / \mathrm{min}$, retention times: $7.9 \mathrm{~min}$ (major isomer) and $14.2 \mathrm{~min}$ (minor isomer).

(+)-5-(Benzoylbenzylamino)-7-methyl-6-(trimethylsilanyl)-1,3-dihydroisobenzofuran-4-carboxylic acid methyl ester (3fa, eq 1).

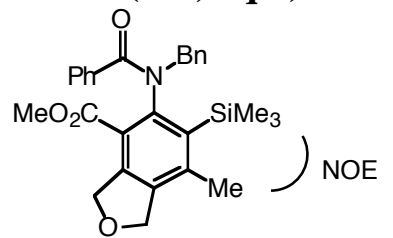

Colorless oil; $[\alpha]_{\mathrm{D}}^{25}+53.5^{\circ}\left(c 0.695, \mathrm{CHCl}_{3}, 50 \%\right.$ ee); IR (neat); 2900, 1700, 1630, 1380, 1260, 840, $700 \mathrm{~cm}^{-1} ;{ }^{1} \mathrm{H}$ NMR $\left(\mathrm{CDCl}_{3}, 300 \mathrm{MHz}\right) \delta 7.36-7.47(\mathrm{~m}, 2 \mathrm{H}), 7.03-7.32$ $(\mathrm{m}, 8 \mathrm{H}), 5.79(\mathrm{~d}, J=13.5 \mathrm{~Hz}, 1 \mathrm{H}), 5.18(\mathrm{~d}, J=14.7 \mathrm{~Hz}, 1 \mathrm{H}), 5.09(\mathrm{~s}, 2 \mathrm{H}), 4.95$ (d, $J=14.7$ $\mathrm{Hz}, 1 \mathrm{H}), 3.98(\mathrm{~d}, J=13.5 \mathrm{~Hz}, 1 \mathrm{H}), 3.20(\mathrm{~s}, 3 \mathrm{H}), 2.43(\mathrm{~s}, 3 \mathrm{H}), 0.41(\mathrm{~s}, 9 \mathrm{H}) ;{ }^{13} \mathrm{C} \mathrm{NMR}$ $\left(\mathrm{CDCl}_{3}, 75 \mathrm{MHz}\right) \delta 168.7,165.0,146.7,143.4,143.0,139.7,139.1,136.1,135.5,130.9$, $129.7,129.5,128.0,127.7,127.3,122.8,75.6,73.2,57.4,51.5,20.8,2.2$; HRMS (ESI) calcd for $\mathrm{C}_{28} \mathrm{H}_{31} \mathrm{NO}_{4} \mathrm{Si}[\mathrm{M}+\mathrm{Na}]^{+}$496.1920, found 496.1923. CHIRALPAK AD-H, hexane:2-PrOH = 90:10, $1.0 \mathrm{~mL} / \mathrm{min}$, retention times: $23.8 \mathrm{~min}$ (minor isomer) and 27.1 min (major isomer). The structure of $\mathbf{3 f a}$ was confirmed by NOE experiments.

\section{References}

1) Duong, H. A.; Cross, M. J.; Louie, J. J. Am. Chem. Soc. 2004, 126, 11438-11439.

2) Atkinson, R. S.; Grimshire, M. J. J. Chem. Soc., Perkin Trans. 1 1986. 7, 1215-1224.

3) Yamamoto, Y.; Nagata, A.; Ando, Y.; Arikawa, Y.; Tatsumi, K.; Itoh, K. Chem. Eur. J. 2003, 9, 2469-2483.

4) Witulski, B.; Stengel, T. Angew. Chem., Int. Ed. 1998, 37, 489-492.

5) Nishikawa, T.; Kakiya, H.; Shinokubo, H.; Oshima, K. J. Am. Chem. Soc. 2001, 123, 4629-4630.

6) Tsukada, N.; Sugawara, S.; Nakaoka, K.; Inoue, Y. J. Org. Chem. 2003, 68, 5961-5966. 

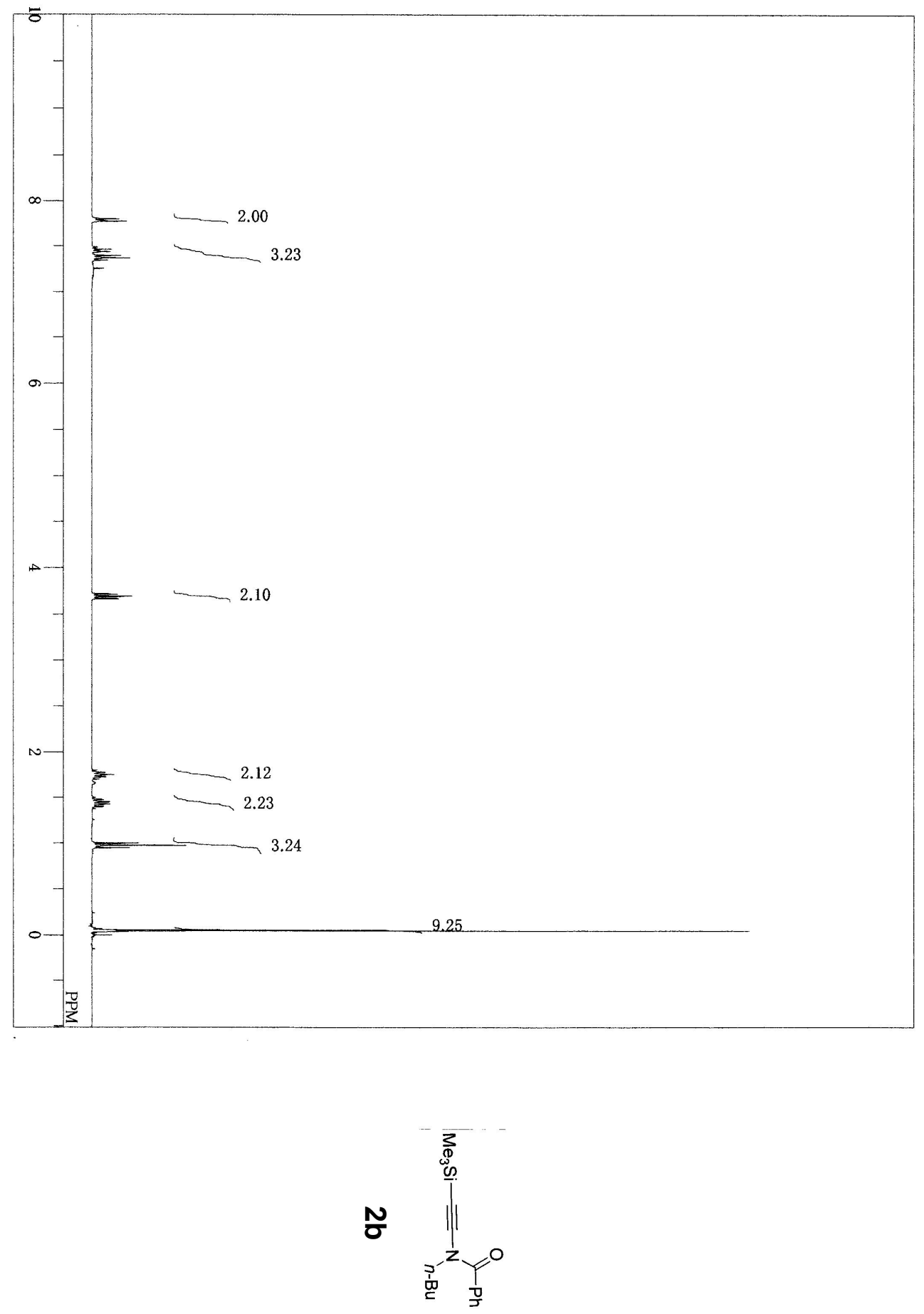

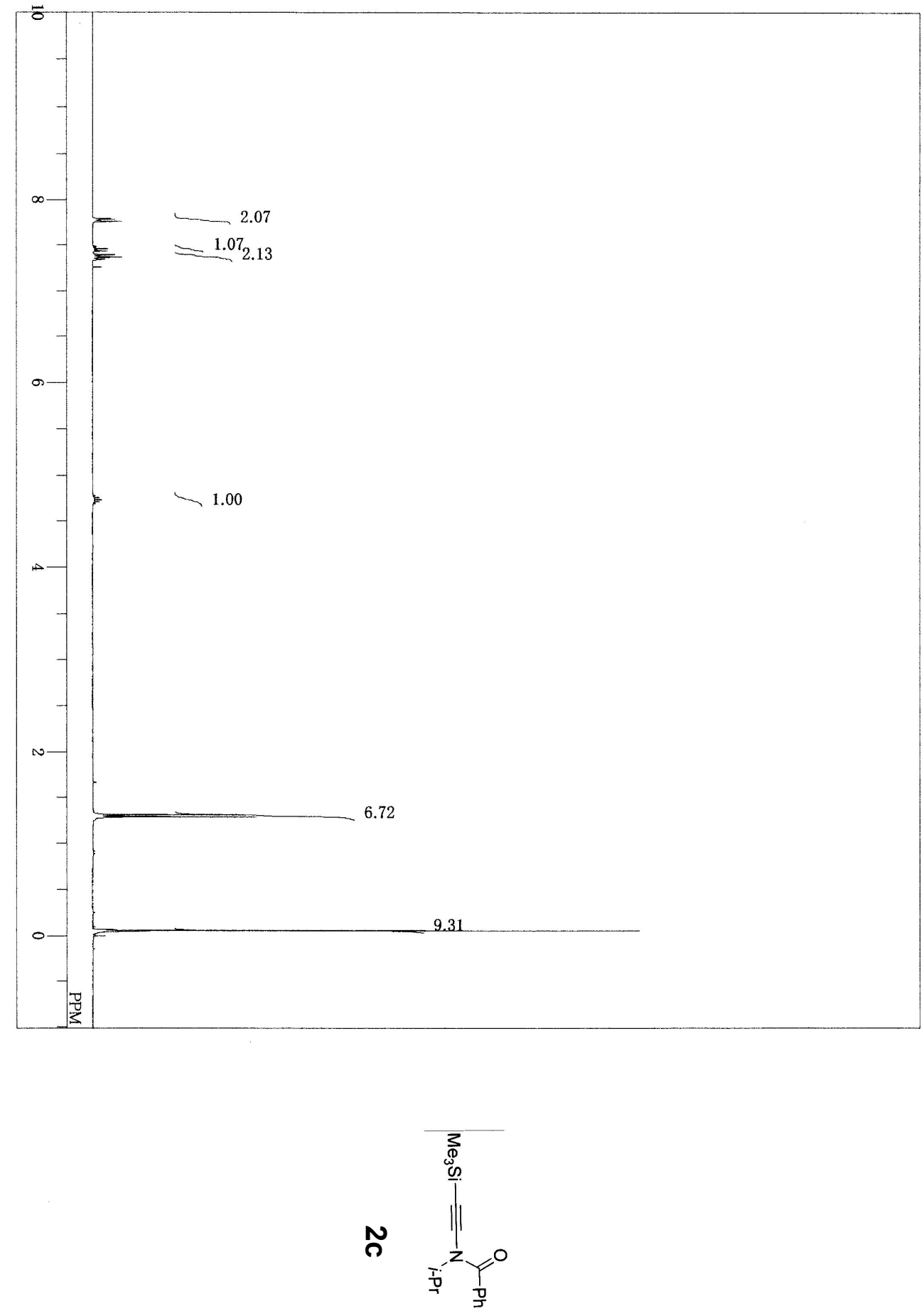

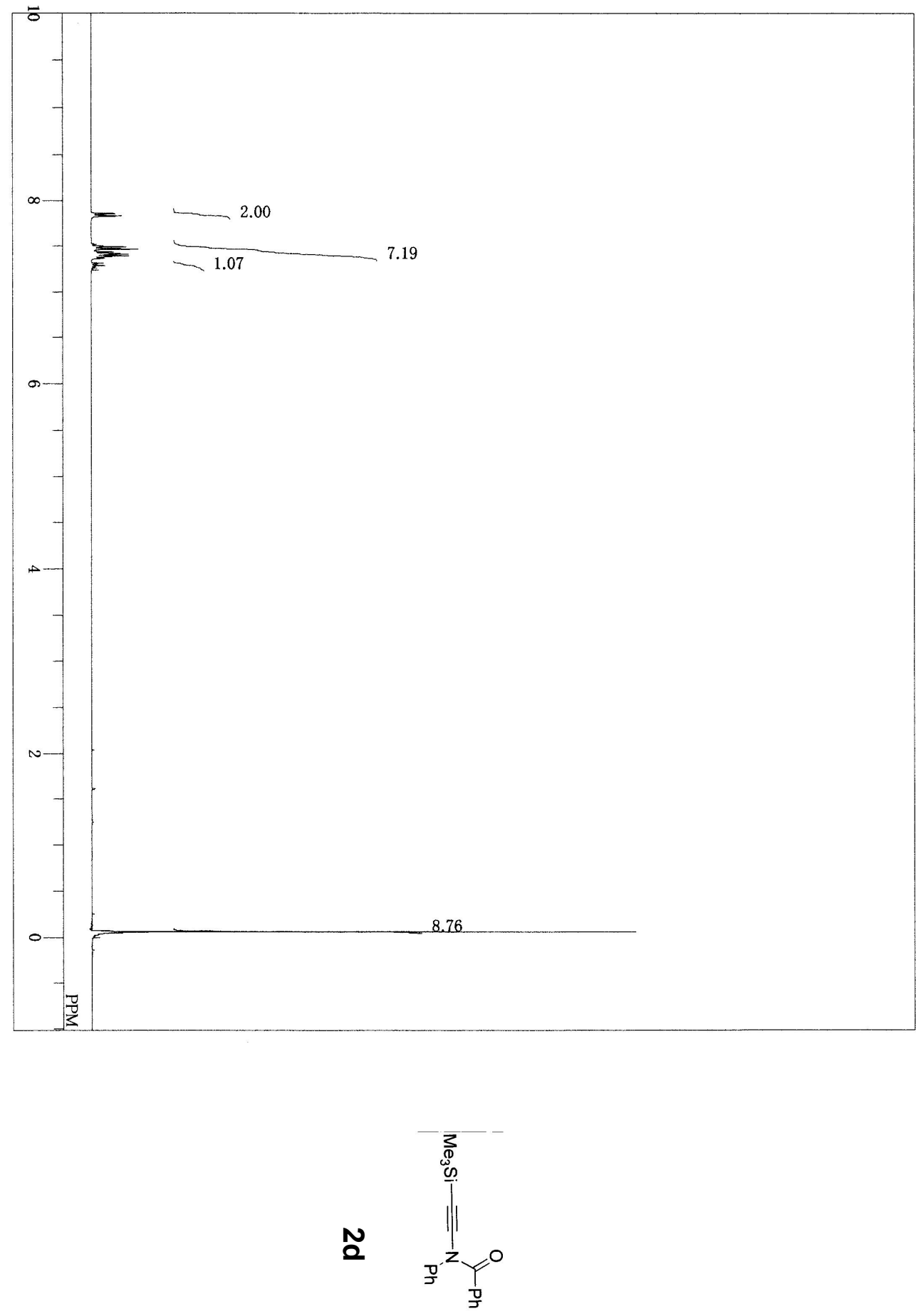

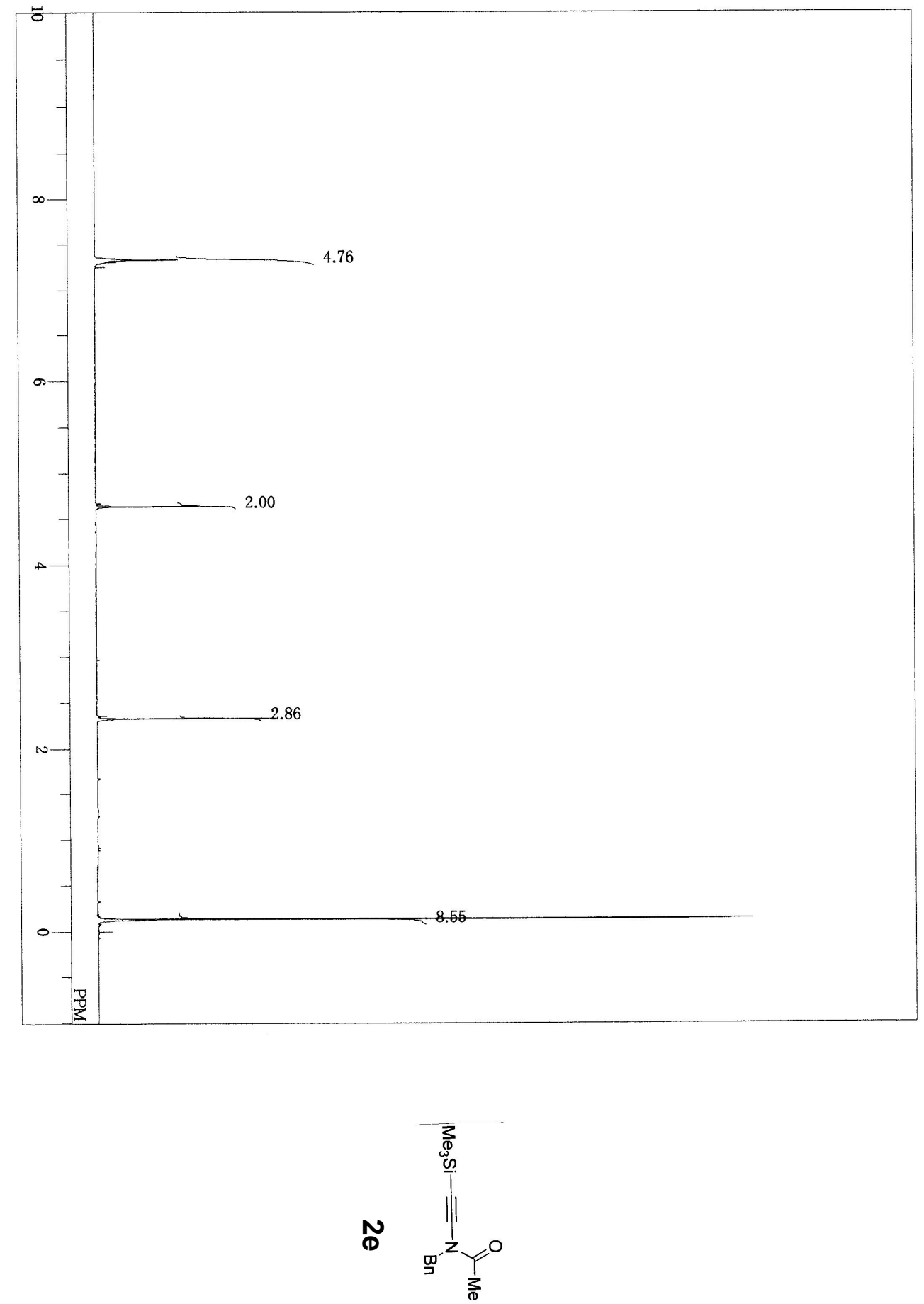

S13 

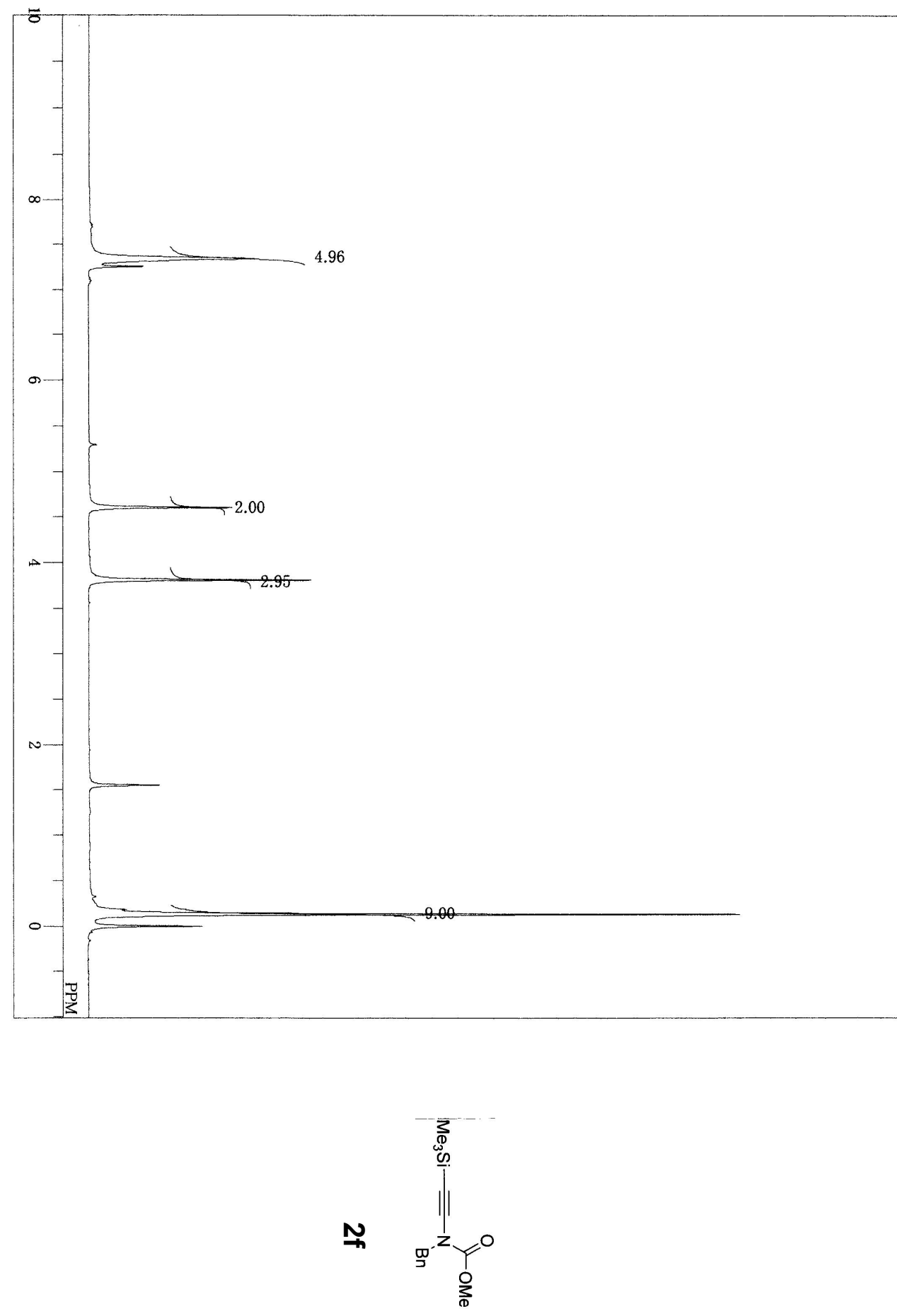

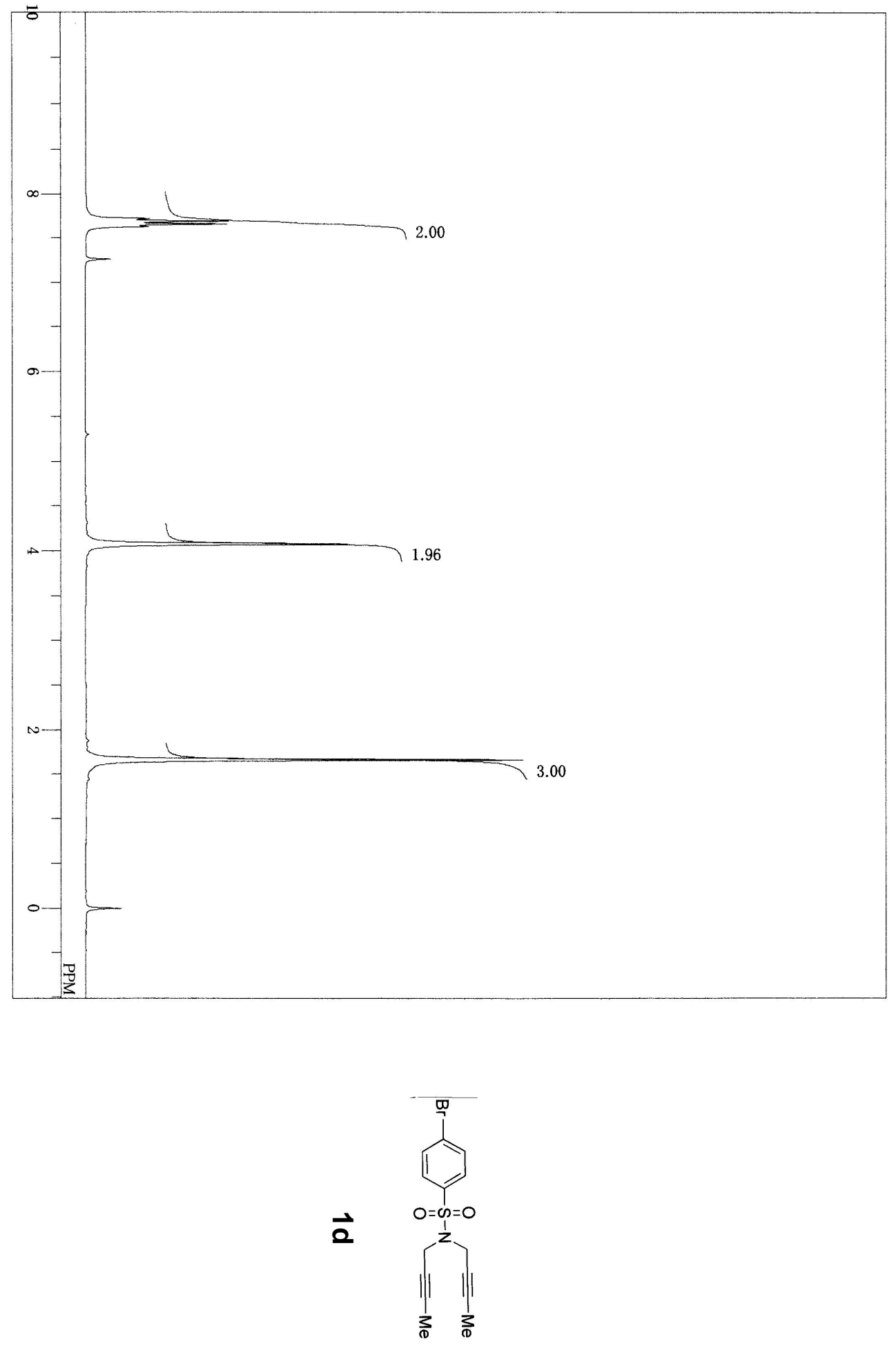

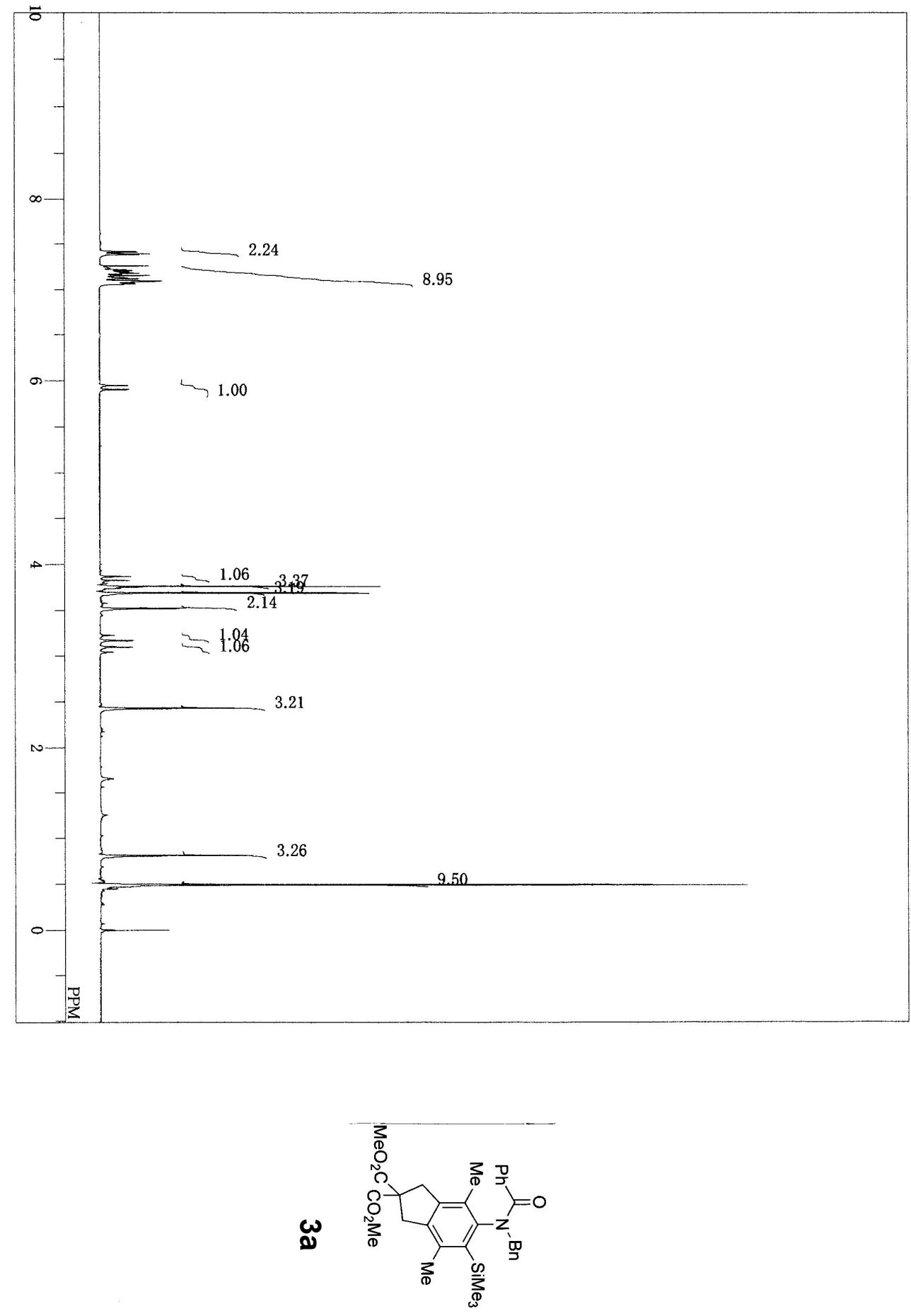

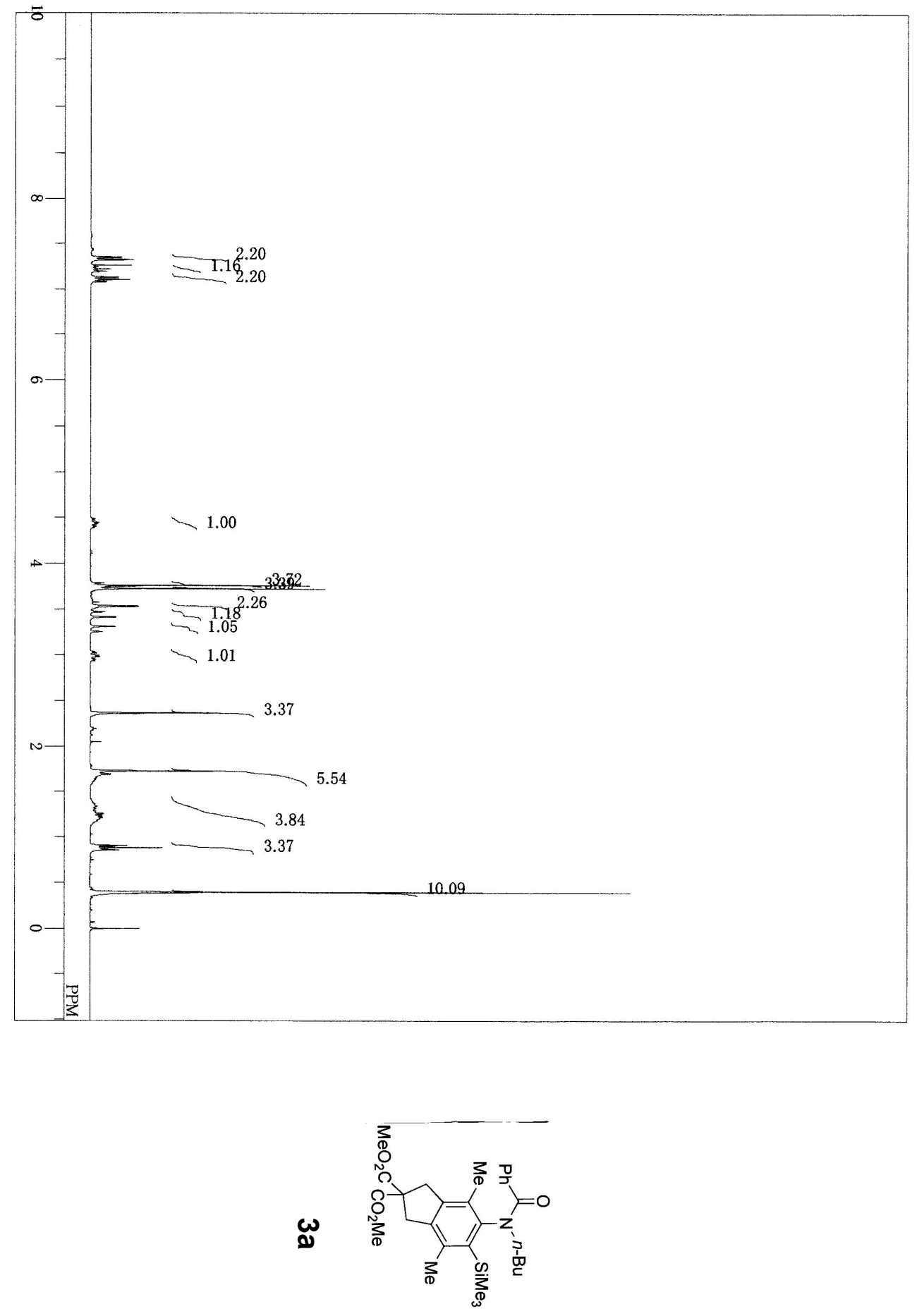

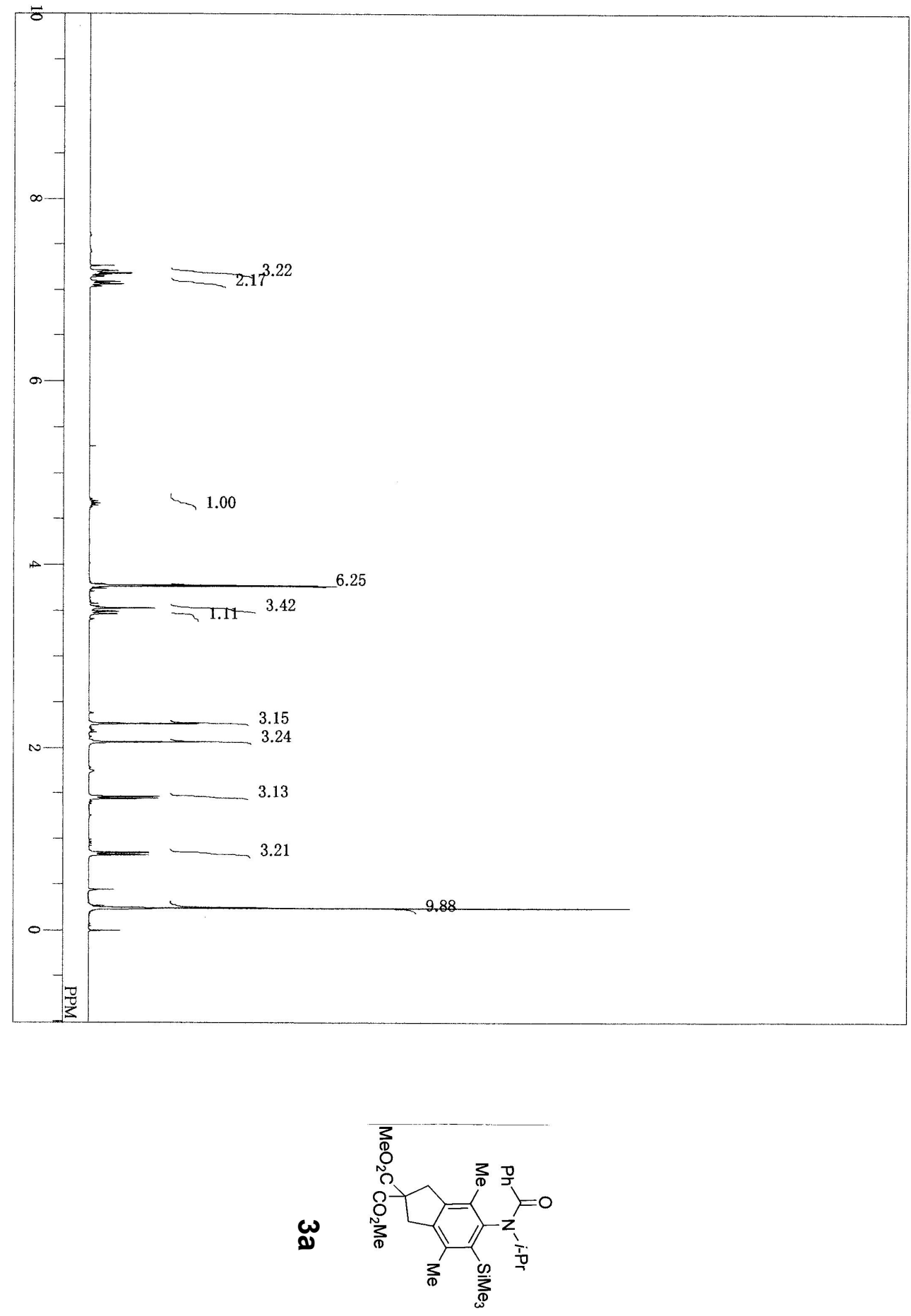

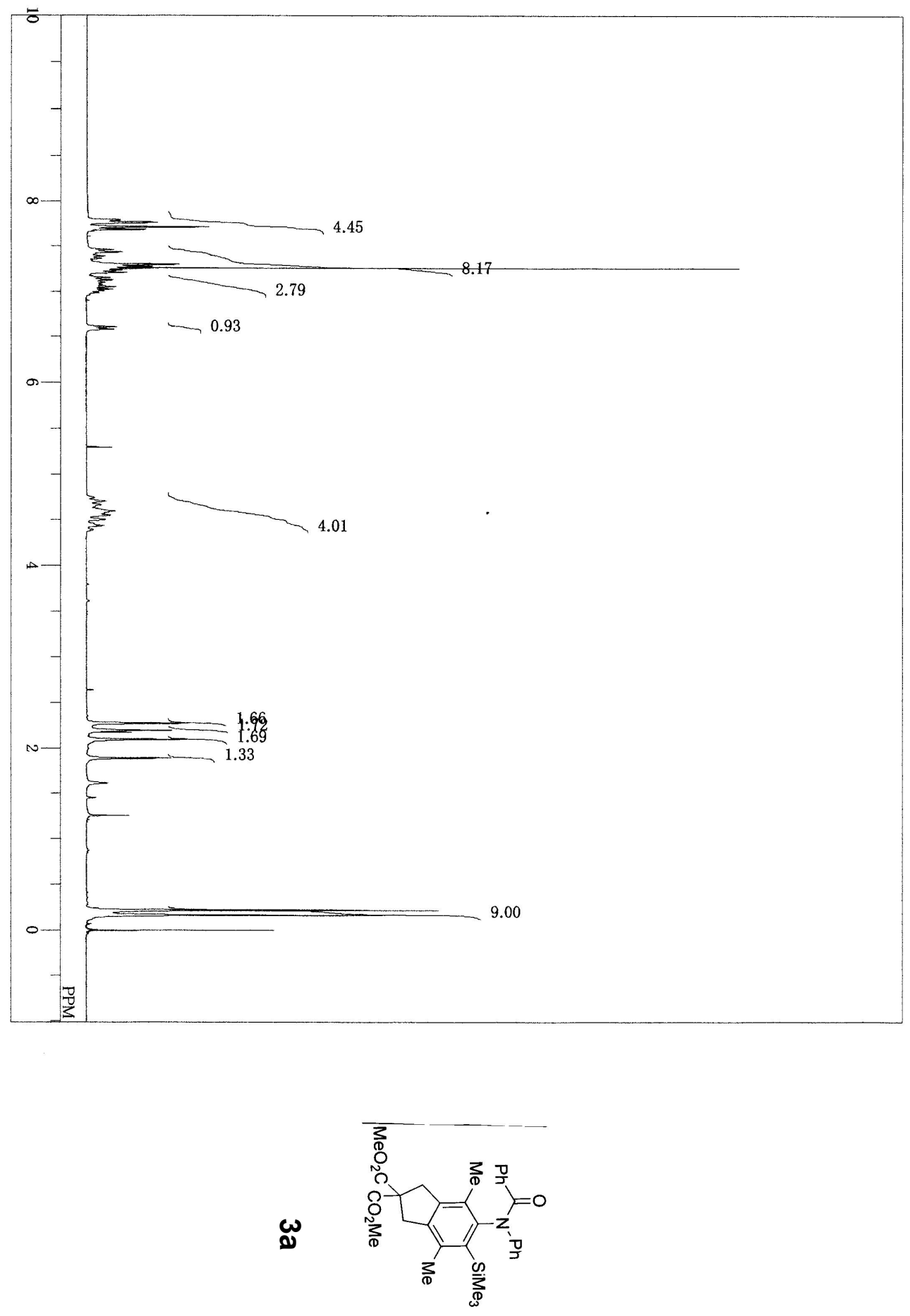

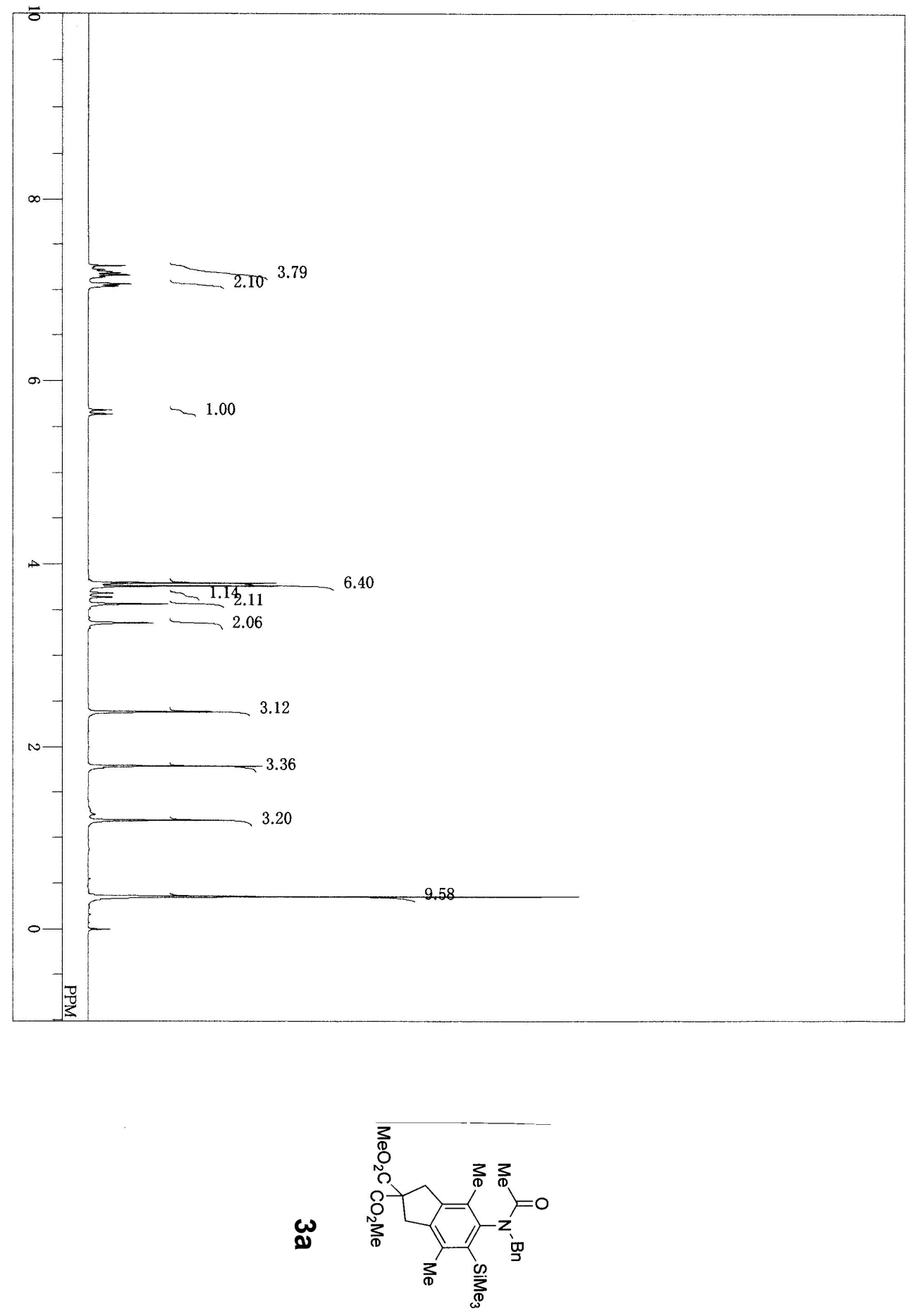

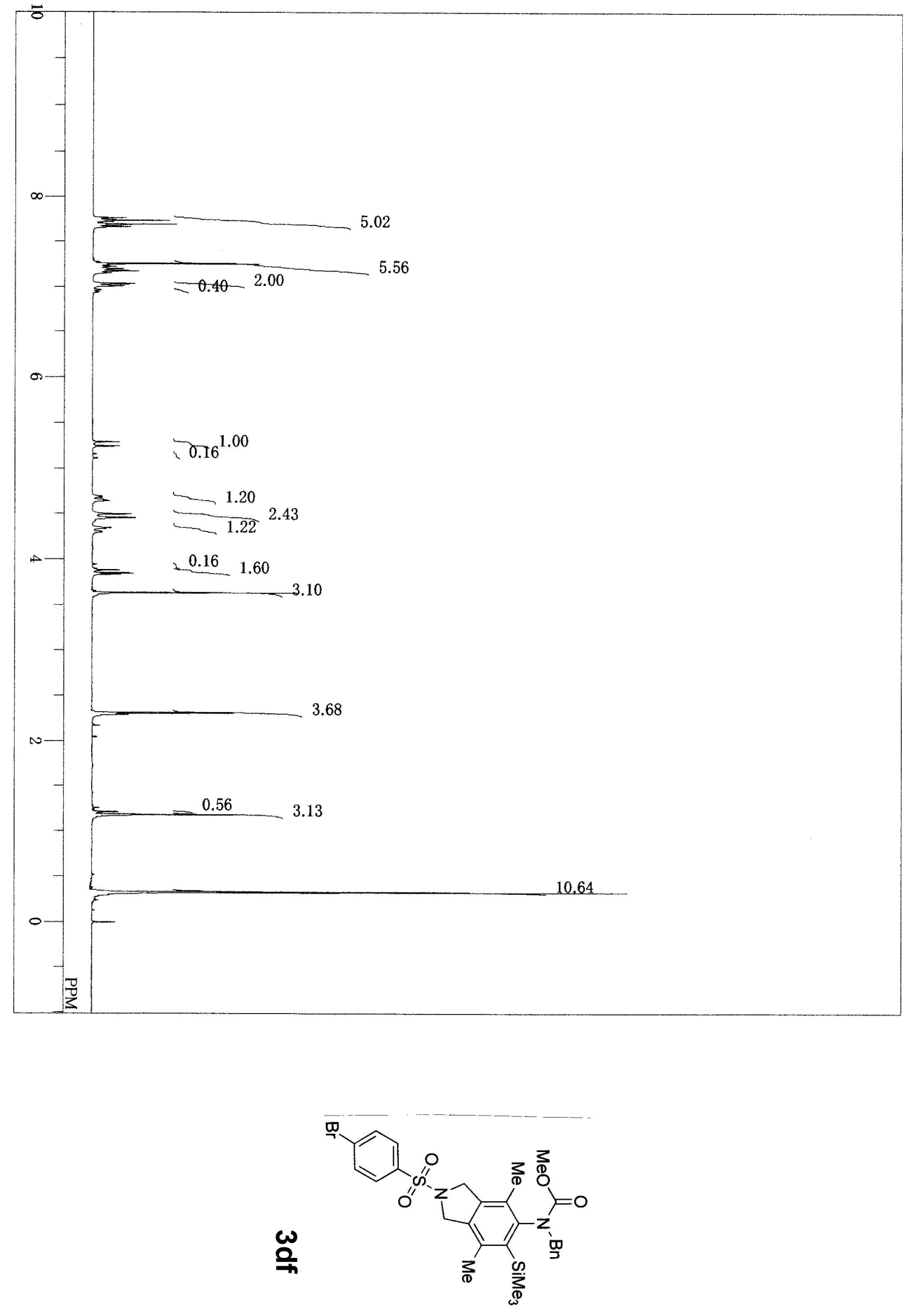

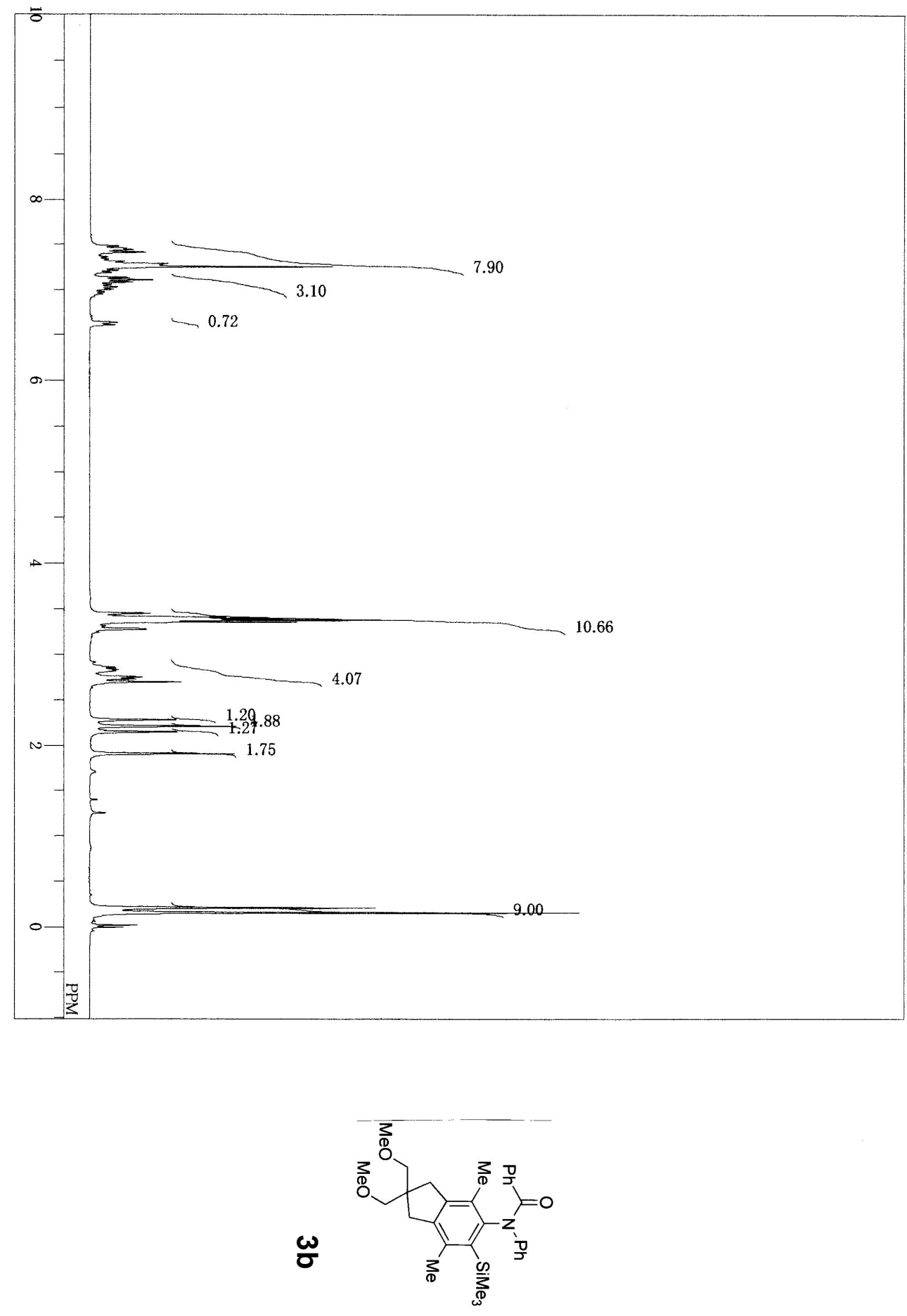

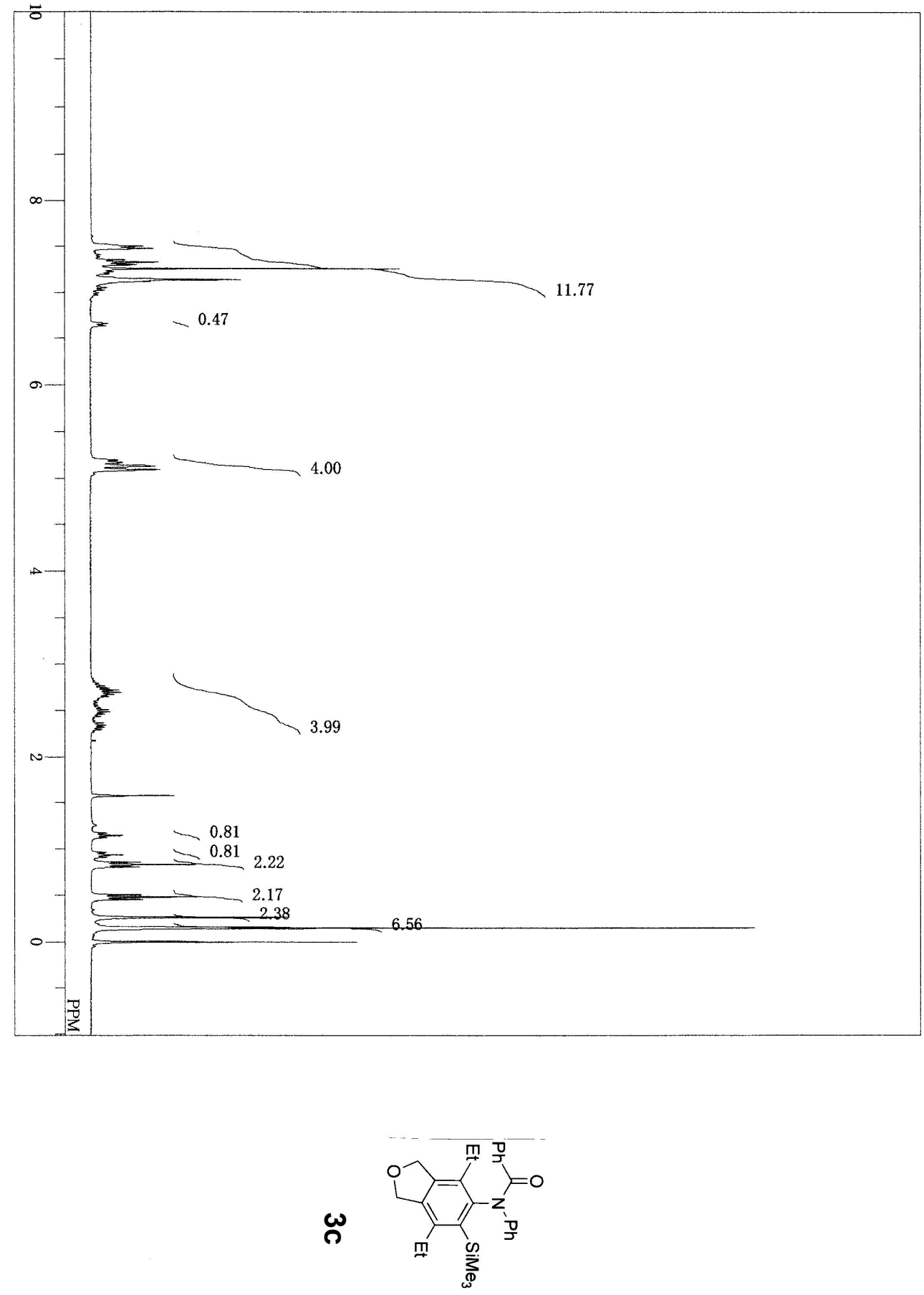

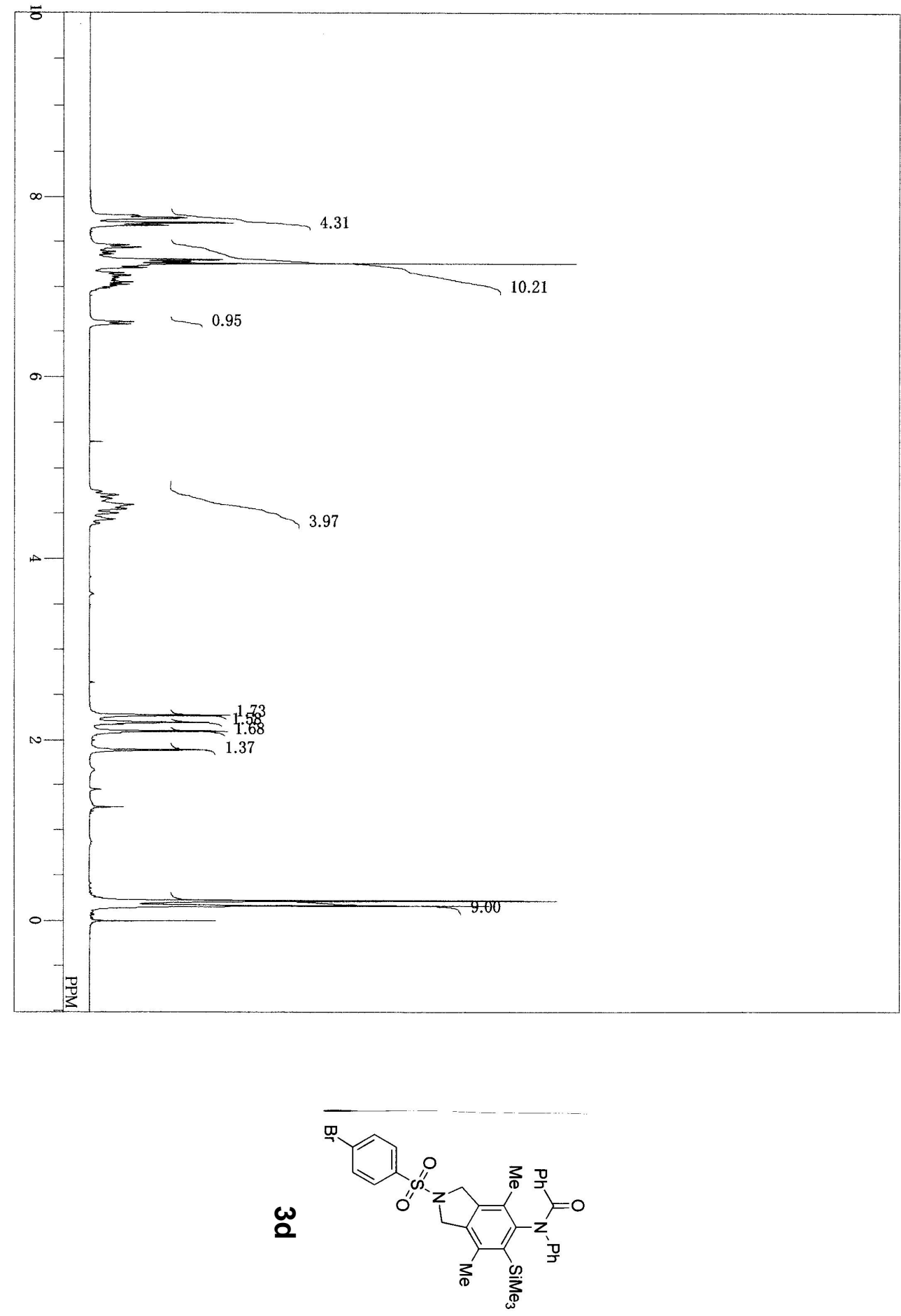

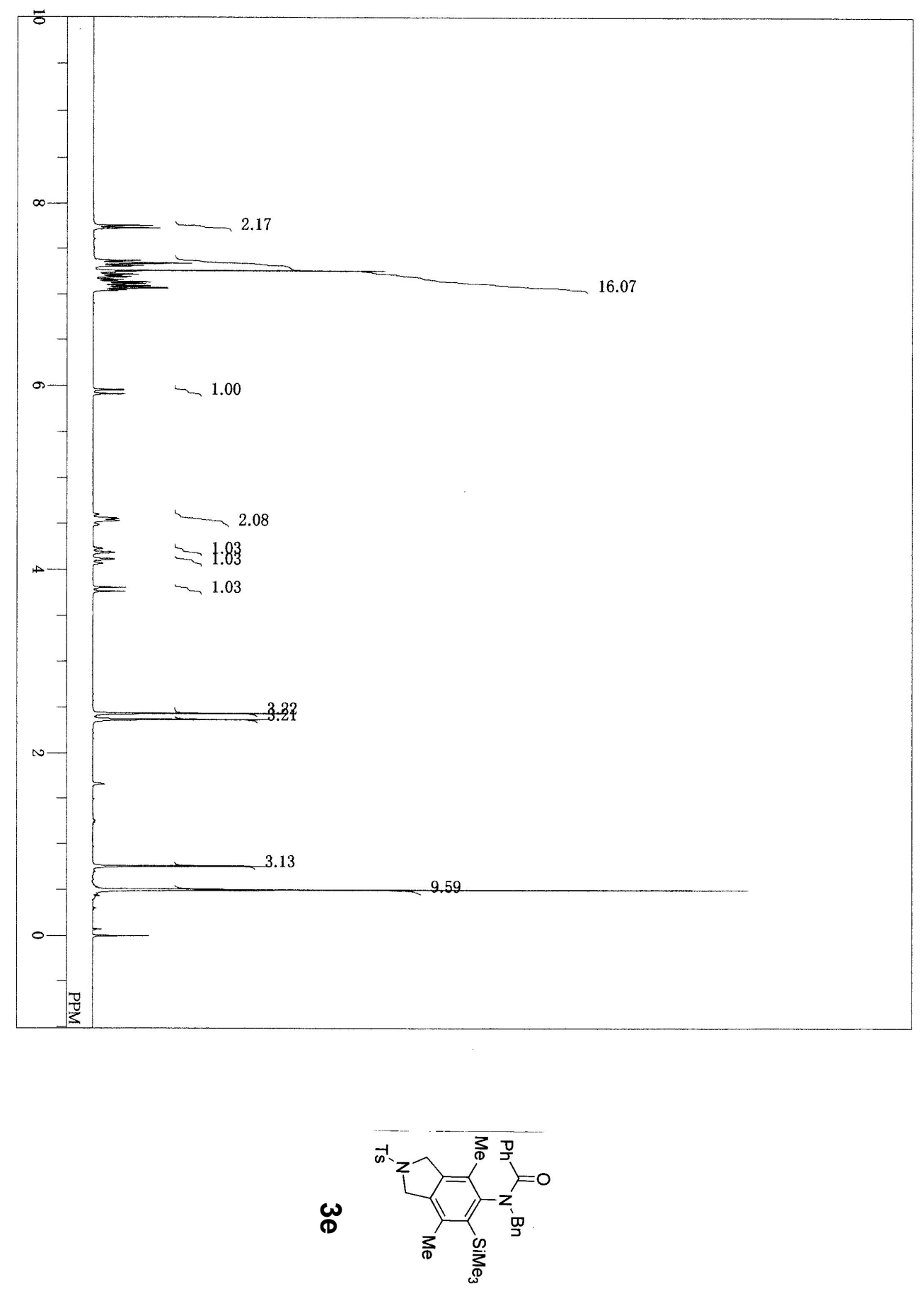

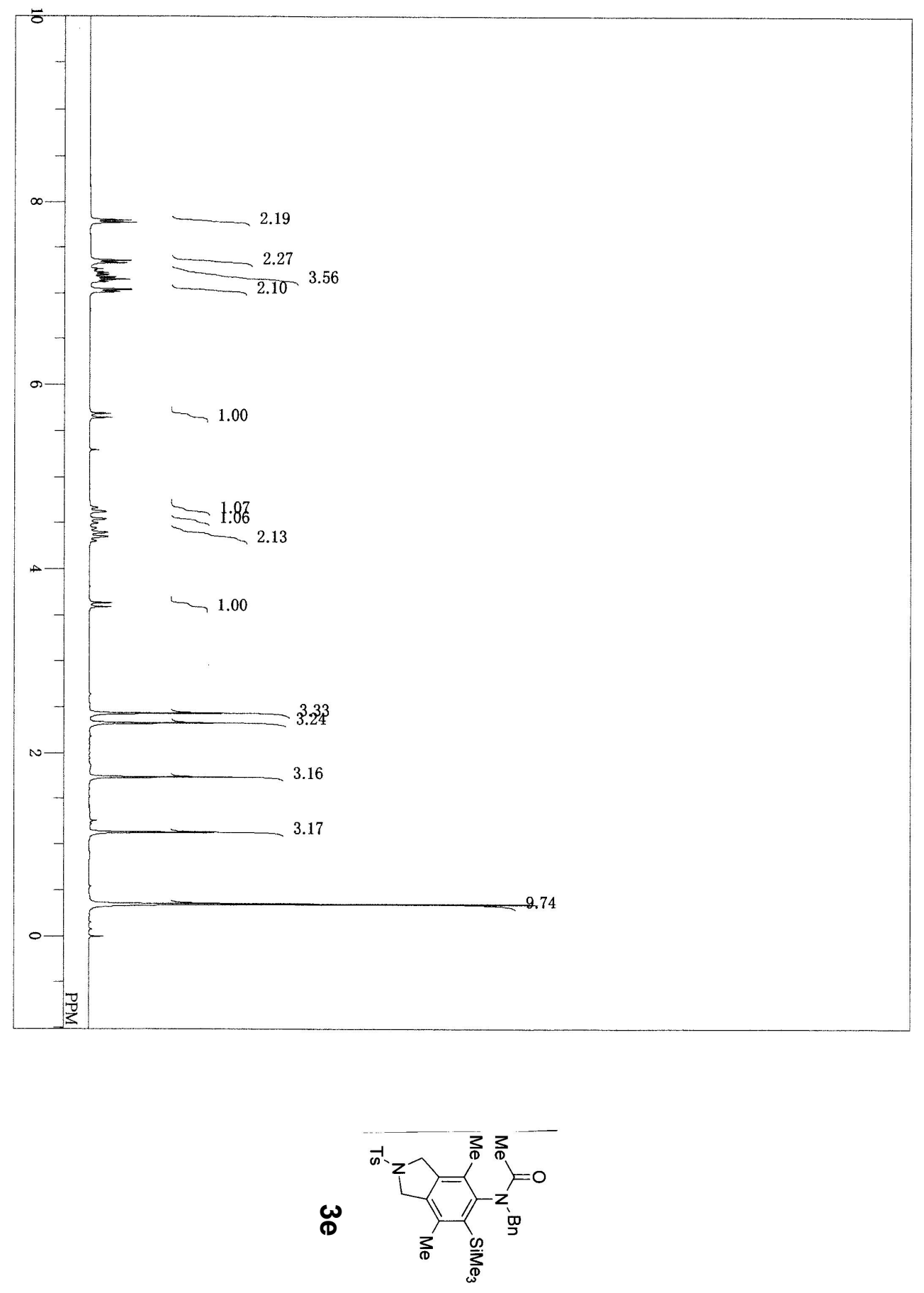

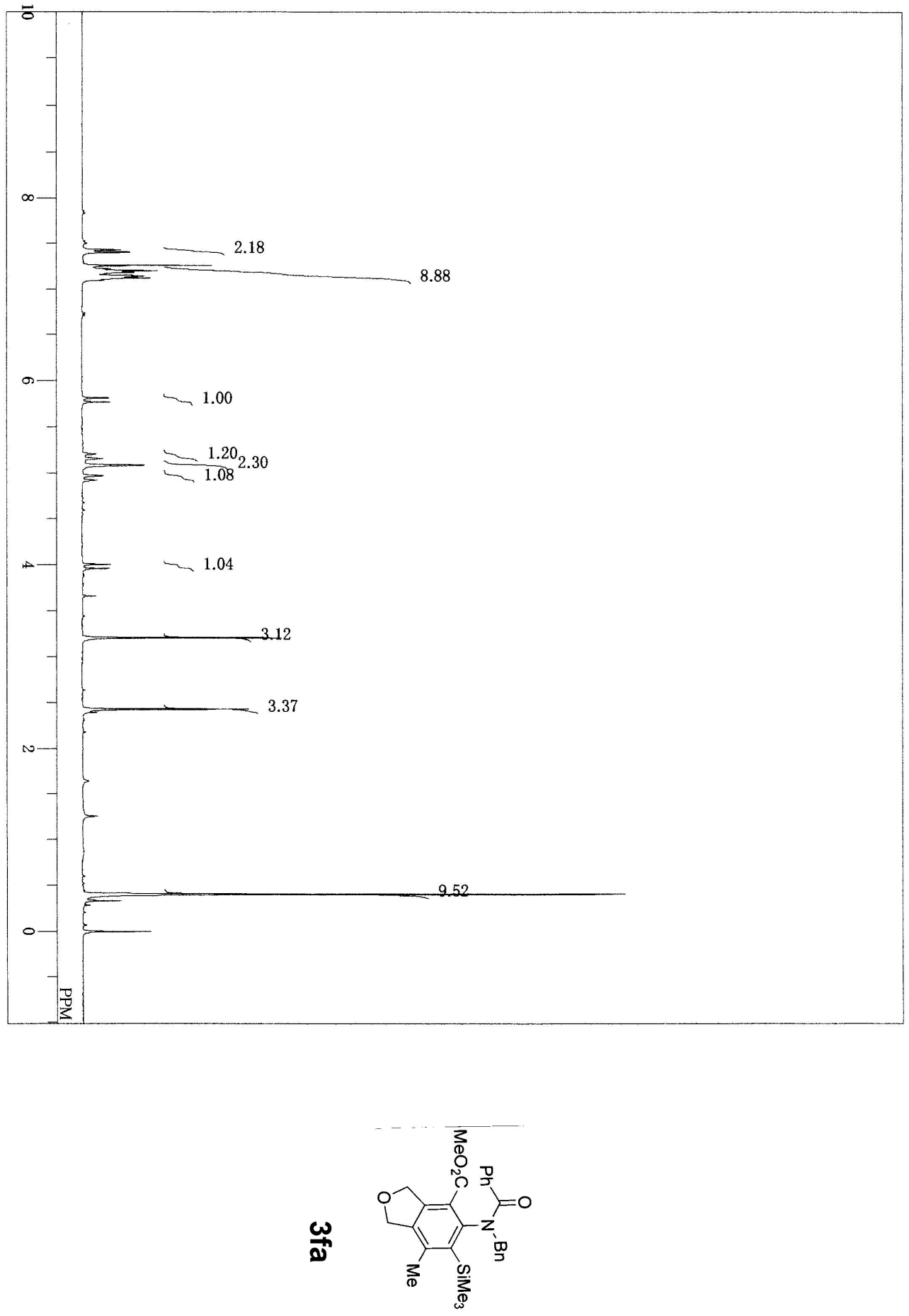